\title{
ON THE BEHAVIOUR OF THE SOLUTIONS TO $p$-LAPLACIAN EQUATIONS AS $p$ GOES TO 1
}

\author{
A. Mercaldo, S. Segura de León, and C. Trombetti
}

Abstract

In the present paper we study the behaviour as $p$ goes to 1 of the weak solutions to the problems

$$
\begin{cases}-\operatorname{div}\left(\left|\nabla u_{p}\right|^{p-2} \nabla u_{p}\right)=f & \text { in } \Omega \\ u_{p}=0 & \text { on } \partial \Omega,\end{cases}
$$

where $\Omega$ is a bounded open set of $\mathbb{R}^{N}(N \geq 2)$ with Lipschitz boundary and $p>1$. As far as the datum $f$ is concerned, we analyze several cases: the most general one is $f \in W^{-1, \infty}(\Omega)$. We also illustrate our results by means of remarks and examples.

\section{Introduction}

In the present paper we study the behaviour, when $p$ goes to 1 , of the solutions $u_{p} \in W_{0}^{1, p}(\Omega)$ to the problems

$$
\begin{cases}-\operatorname{div}\left(\left|\nabla u_{p}\right|^{p-2} \nabla u_{p}\right)=f & \text { in } \Omega \\ u_{p}=0 & \text { on } \partial \Omega,\end{cases}
$$

where $p>1$ and $\Omega$ is a bounded open set of $\mathbb{R}^{N}(N \geq 2)$ with Lipschitz boundary. We analyze the case where $\Omega$ is a ball and the datum $f$ is a non-negative radially decreasing function belonging to the Lorentz space $L^{N, \infty}(\Omega)$ and the case where the datum $f$ belongs to the dual space $W^{-1, \infty}(\Omega)$.

We are interested in finding the pointwise limit of $u_{p}$ as $p$ goes to 1 and in proving that such a limit is a solution to the "limit equation" of (1.1), namely:

$$
-\operatorname{div}\left(\frac{D u}{|D u|}\right)=f \quad \text { in } \Omega
$$

2000 Mathematics Subject Classification. 35J20, 35J70.

Key words. Nonlinear elliptic equations, 1-Laplace operator. 
with homogeneous Dirichlet boundary condition. Hence, firstly we study the behaviour of $u_{p}$ when $p$ goes to 1 , finding a limit function $u$, and secondly we prove that such a limit function $u$ is a solution to (1.2). Both aspects of our study have been investigated by several authors. The interest in studying the behaviour of $u_{p}$ comes from an optimal design problem in the theory of torsion and related geometrical problems (see [16] and [17]). The behaviour of $u_{p}$ in the case where the datum $f$ is constant has been studied in [16] by Kawohl, where the author proved that under a suitable smallness assumption on the domain, it results

$$
\lim _{p \rightarrow 1} u_{p}=0,
$$

while under the assumption that the domain is large enough one has

$$
\lim _{p \rightarrow 1} u_{p}=+\infty .
$$

The behaviour of $u_{p}$ in the case where $f$ is not constant and it belongs to the Lebesgue space $L^{N}(\Omega)$ (or to the Lorentz space $L^{N, \infty}(\Omega)$ ) is studied in [10]. In such a paper the authors prove again (1.3) under the assumption that the $L^{N}(\Omega)$-norm (or $L^{N, \infty}(\Omega)$-norm) of the datum $f$ is small enough.

As just pointed out, the second aim of our study consists in proving that the limit function $u=\lim _{p \rightarrow 1} u_{p}$ is a solution to problem (1.2). The limit equation (1.2) have been studied by various authors (see for instance $[\mathbf{4}],[\mathbf{6}],[\mathbf{9}],[\mathbf{1 1}],[\mathbf{1 2}]$ and references there in). Motivations for such an interest are found in the variational approach to image restoration introduced by L. Rudin, S. Osher and E. Fatemi ${ }^{1}$. The definitions of solutions to equation (1.2) typically consider a datum in $L^{N}(\Omega)$ or $L_{\text {loc }}^{N}(\Omega)$; moreover such solutions are functions belonging to the space $B V(\Omega)$, which guaranties the existence of a distributional gradient, well defined as a Radon measure. In order to give a meaning to $\frac{D u}{|D u|}$ in the limit equation, any definition of solution to (1.2) relies on the existence of a vector field $z: \Omega \rightarrow \mathbb{R}^{N}$, which belongs to $L^{\infty}\left(\Omega ; \mathbb{R}^{N}\right)$, with $\|z\|_{\infty} \leq 1$. Moreover $z$ satisfies the equation $-\operatorname{div} z=f$ in the distributional sense and $z \cdot D u=|D u|$. The boundary condition may be included as $z \cdot \nu \in \operatorname{sign}(-u)$ a.e. on $\partial \Omega$ where $\nu$ denotes the outward normal unit vector. The expressions $z \cdot D u$ and $z \cdot \nu$ have sense thanks to the Anzellotti theory (see [7] or [5]) which defines a Radon measure $(z, D u)$,

\footnotetext{
${ }^{1}$ For a review on the development of variational models in image processing and a
} deep study of the Minimizing Total Variation Flow, see [5]. 
provides the definition of a weakly trace on $\partial \Omega$ to the normal component of $z$, denoted by $[z, \nu]$, and guaranties a Green's formula. Roughly speaking, $z$ plays the role of $\frac{D u}{|D u|}$.

In this paper we consider problem (1.2) with data belonging to the Lorentz space $L^{N, \infty}(\Omega)$ and to the dual space $W^{-1, \infty}(\Omega)$. Let us now explain the reason for which we consider such types of data. The embedding $W^{-1, \infty}(\Omega) \hookrightarrow W^{-1, p^{\prime}}(\Omega)$ for all $p>1$ ensures the existence of an unique weak solution $u_{p} \in W_{0}^{1, p}(\Omega)$ to problem (1.1) (see [18]). Smallness assumption on the data allows us to prove the existence of a limit function $u=\lim _{p \rightarrow 1} u_{p}$ which belongs to the space $B V(\Omega)$. On the other hand, we prove the existence of a vector field $z \in L^{\infty}\left(\Omega, \mathbb{R}^{N}\right)$ satisfying $-\operatorname{div} z=f$ in the sense of distributions. This implies that

$$
|\langle f, \varphi\rangle|=\left|\int_{\Omega} z \cdot \nabla \varphi d x\right| \leq\|z\|_{\infty} \int_{\Omega}|\nabla \varphi| d x
$$

for all $\varphi \in C_{0}^{\infty}(\Omega)$, and hence $f \in W^{-1, \infty}(\Omega)$.

Observe that we take a datum belonging to $W^{-1, \infty}(\Omega)$ and we find a solution in $B V(\Omega)$, even if $W^{-1, \infty}(\Omega)$ is not the dual space of $B V(\Omega)$. This fact yields some difficulties which we completely solve only in the case where the datum $f$ belongs to specific subspaces of $W^{-1, \infty}(\Omega)$.

Let us observe that, by the improvement of Sobolev embedding (see for example [1] ) and duality arguments, the Lorentz space $L^{N, \infty}(\Omega)$ is a subset of the dual space $W^{-1, \infty}(\Omega)$. We will consider data belonging to $L^{N, \infty}(\Omega)$ which are radially symmetric, without smallness assumptions. Indeed such symmetries allow us to write the expression of the solutions $u_{p}$ and to handle it in order to study the behaviour of $u_{p}$.

The case where the data do not belong to $W^{-1, \infty}(\Omega)$ have been considered in [4], [6], [9]. However, in such papers the solutions to the "limit equation" (1.2) are not obtained as limit of $u_{p}$, except in the case where the data are smooth enough, and the methods employed do not apply in our framework. We explicitly remark that the asymptotic behaviour of $u_{p}$ when the datum $f$ belongs to $L^{1}(\Omega)$ will be studied by the authors in the forthcoming paper [19], where a notion of solution to the equation (1.2) is introduced.

Finally we summarize the contents of the present paper. After introducing our notation (see Section 2), we begin by studying the case where $f$ is a radially decreasing function defined in a ball $\Omega$ and belonging to $L^{N, \infty}(\Omega)$, without assuming any smallness condition on its $L^{N, \infty}(\Omega)$-norm (see Section 3). Next we study the case where $f$ belongs to the dual space $W^{-1, \infty}(\Omega)$ and we prove that (1.3) holds true under the 
assumption $\|f\|_{W^{-1, \infty}(\Omega)}<1$. We also prove that if $\|f\|_{W^{-1, \infty}(\Omega)}=1$, then $u_{p}$ tends to a $B V$-function, and if $\|f\|_{W^{-1, \infty}(\Omega)}>1$, then there is not any $B V$-function which is the pointwise limit of $u_{p}$ (see Section 4). In general we are not able to prove that $u_{p}$ tends to a solution to problem (1.2) when $\|f\|_{W^{-1, \infty}(\Omega)}=1$. In such a case we have to assume that $f$ belongs to the predual space of $B V(\Omega)$ (see Subsection 4.2).

We conclude with few words on the Appendix. First we present some properties of the predual space of $B V(\Omega)$ and we prove that its norm as a subspace of the dual of $B V(\Omega)$ is exactly the same as the norm of $W^{-1, \infty}(\Omega)$. Secondly, we adapt Anzellotti's theory in the framework of the predual of $B V(\Omega)$.

\section{Notation}

In this section we will introduce some notation which will be used throughout this paper. We will denote by $\Omega$ a bounded open subset of $\mathbb{R}^{N}$ with Lipschitz boundary. Thus there exists a unit vector field (denoted by $\nu$ ) normal to $\partial \Omega$ and exterior to $\Omega$, defined $\mathcal{H}^{N-1}$-a.e. on $\partial \Omega$. Here $\mathcal{H}^{N-1}$ denotes the $(N-1)$-dimensional Hausdorff measure. Here and in the sequel, $|E|$ denotes the Lebesgue measure of a measurable subset $E$ of $\mathbb{R}^{N}$.

Let $u: \Omega \rightarrow \mathbb{R}$ be a measurable function. We denote by $\mu_{u}$ the distribution function of $u$, that is the function $\mu_{u}:[0,+\infty[\rightarrow[0,+\infty[$ defined by

$$
\mu_{u}(t)=|\{x \in \Omega:|u(x)|>t\}|, \quad t \geq 0 .
$$

The decreasing rearrangement of $u$ is the function $\left.\left.u^{*}:\right] 0,|\Omega|\right] \rightarrow \mathbb{R}^{+}$ defined by

$$
\left.\left.u^{*}(s)=\sup \left\{t>0: \mu_{u}(t)>s\right\}, \quad s \in\right] 0,|\Omega|\right] .
$$

For $1<q<\infty$, the Lorentz space $L^{q, \infty}(\Omega)$, also known as Marcinkiewicz or weak-Lebesgue, is the space of Lebesgue measurable functions $u$ such that

$$
\sup _{t>0} t \mu_{u}(t)^{1 / q}<+\infty
$$

It is endowed with the norm

$$
\|u\|_{q, \infty}=\sup _{0<s<|\Omega|} s^{\frac{1}{q}} u^{* *}(s),
$$


where $u^{* *}(s)=\frac{1}{s} \int_{0}^{s} u^{*}(\sigma) d \sigma$. For $1<q<\infty$, the Lorentz space $L^{q, 1}(\Omega)$ is the space of all Lebesgue measurable functions $u$ such that

$$
\|u\|_{q, 1}=\int_{0}^{\infty} t^{\frac{1}{q}-1} u^{*}(t) d t<+\infty
$$

endowed with the norm (2.2). It is well-known (cf. [15], [21]) that the following inclusions hold

$$
L^{q+\epsilon}(\Omega) \hookrightarrow L^{q, 1}(\Omega) \hookrightarrow L^{q}(\Omega) \hookrightarrow L^{q, \infty}(\Omega) \hookrightarrow L^{q-\epsilon}(\Omega),
$$

for every $\epsilon>0$. Finally we recall that the Marcinkiewicz space $L^{N, \infty}(\Omega)$ is the dual space of $L^{\frac{N}{N-1}}, 1(\Omega)$.

We define $\mathcal{M}(\Omega)$ as the space of all Radon measures with bounded total variation on $\Omega$ and we denote by $|\mu|$ the total variation of $\mu \in$ $\mathcal{M}(\Omega)$. The space of all functions of finite variation, that is the space of those $u \in L^{1}(\Omega)$ whose distributional derivatives belong to $\mathcal{M}(\Omega)$, is denoted by $B V(\Omega)$. It is endowed with the norm defined by $\|u\|_{B V(\Omega)}=$ $\int_{\Omega}|u| d x+|D u|(\Omega)$, for any $u \in B V(\Omega)$. Since $\Omega$ has Lipschitz boundary, if $u$ belongs to $B V(\Omega)$, then the function

$$
u_{0}= \begin{cases}u, & \text { in } \Omega ; \\ 0, & \text { in } \mathbb{R}^{N} \backslash \Omega ;\end{cases}
$$

belongs to $B V\left(\mathbb{R}^{N}\right)$ and $\left|D u_{0}\right|\left(\mathbb{R}^{N}\right)=\int_{\partial \Omega}|u| d \mathcal{H}^{N-1}+|D u|(\Omega)$. We explicitly point out that $\left|D u_{0}\right|\left(\mathbb{R}^{N}\right)$ defines an equivalent norm on $B V(\Omega)$, which we will use in the sequel. Through the paper, with an abuse of notation, we still denote $u_{0}$ by $u$.

We will denote by $S_{N, p}$ the best constant in the Sobolev inequality (cf. [22]), that is,

$$
\|u\|_{p^{*}} \leq S_{N, p}\|\nabla u\|_{p}, \quad \text { for all } u \in W_{0}^{1, p}(\Omega) .
$$

We will also write $S_{N}$ instead of $S_{N, 1}$. It is well-known (cf. [22]), that

$$
\lim _{p \rightarrow 1} S_{N, p}=S_{N}
$$

Sobolev's inequality can be improved in the context of Lorentz spaces (cf. [1]) and, furthermore, by an approximation argument may be extended to $B V$-functions (see for instance [25]); as a consequence we obtain the continuous embedding

$$
B V(\Omega) \hookrightarrow L^{\frac{N}{N-1}, 1}(\Omega) .
$$


We will denote by $W^{-1, q^{\prime}}(\Omega)$ the dual space of $W_{0}^{1, q}(\Omega), 1 \leq q<\infty$. Here we just recall that the norm in $W^{-1, \infty}(\Omega)$ is given by

$$
\|\mu\|_{W^{-1, \infty}(\Omega)}=\sup \left\{\langle\mu, \varphi\rangle_{W^{-1, \infty}(\Omega), W_{0}^{1,1}(\Omega)}: \int_{\Omega}|\nabla \varphi| d x \leq 1\right\} .
$$

It is worth pointing out some remarkable subspaces of $W^{-1, \infty}(\Omega)$. One of these is $\mathcal{M}(\Omega) \cap W^{-1, \infty}(\Omega)$ whose elements are named Guy David measures in [20]. Another subspace is the so-called predual of $B V(\Omega)$. Indeed, the space $B V(\Omega)$ is the dual of a separable space which will be denoted by $\Gamma(\Omega)$; its elements can be written as $f-\operatorname{div} F$, with $(f, F) \in C_{0}\left(\Omega ; \mathbb{R}^{N+1}\right)$ (see [14], and also $[\mathbf{2 0}]$ and $[\mathbf{3}]$ ). Since the elements of $W^{-1, \infty}(\Omega)$ may be written as $f-\operatorname{div} F$, with $(f, F) \in L^{\infty}\left(\Omega ; \mathbb{R}^{N+1}\right)$, we deduce that $\Gamma(\Omega) \subset W^{-1, \infty}(\Omega)$. Recall that in the 1-dimensional case we have

$$
\begin{gathered}
W^{-1, \infty}(a, b)=\left\{f^{\prime}: f \in L^{\infty}(a, b)\right\} \\
\mathcal{M}(a, b) \cap W^{-1, \infty}(\Omega)=\left\{f^{\prime}: f \in B V(a, b)\right\} \\
\Gamma(a, b)=\left\{f^{\prime}: f \in C(a, b) \text { and } f(a)=f(b)=0\right\} .
\end{gathered}
$$

So that it is easy to find examples in any dimension that show all these spaces are different.

Moreover, we will denote by $B V(\Omega)^{*}$ the dual space of $B V(\Omega)$. The norm in $B V(\Omega)^{*}$ is given by

$\|\mu\|_{B V(\Omega)^{*}}=\sup \left\{\langle\mu, \varphi\rangle_{B V(\Omega)^{*}, B V(\Omega)}:|D \varphi|(\Omega)+\int_{\partial \Omega}|\varphi| d \mathcal{H}^{N-1} \leq 1\right\}$.

Of course, $\Gamma(\Omega) \hookrightarrow B V(\Omega)^{*}$; we will prove in the Appendix below that in the space $\Gamma(\Omega)$ the norms as subset of $B V(\Omega)^{*}$ and as subset of $W^{-1, \infty}(\Omega)$ coincide.

Finally we recall that a sequence $\left(\mu_{n}\right)_{n}$ in $\mathcal{M}(\Omega)$ weakly* converges to $\mu$ if

$$
\lim _{n \rightarrow \infty} \int_{\Omega} f d \mu_{n}=\int_{\Omega} f d \mu
$$

for every $f \in C_{0}(\Omega)$. We will say that a sequence $\left(u_{n}\right)_{n}$ weakly* converges to $u$ in $B V(\Omega)$ if it strongly converges in $L^{1}(\Omega)$ and $\left(D u_{n}\right)_{n}$ weakly* converges to $D u$ in $\mathcal{M}(\Omega)$. At least for sufficiently regular domains, this notion corresponds to weak* convergence in the usual sense: that is with respect to $\sigma(B V(\Omega), \Gamma(\Omega))$. 


\section{The radial case}

In this section we consider problem (1.1) in the case where the domain $\Omega$ is a ball centered at the origin, i.e. $\Omega \equiv B_{R}=\left\{x \in \mathbb{R}^{N}:|x|<R\right\}$ and the datum $f$ is a nonnegative radially decreasing function belonging to the Lorentz space $L^{N, \infty}\left(B_{R}\right)$. Since both the domain and the datum are radially symmetric, it is well-known (see for instance $[\mathbf{2 3}]$ ) that the weak solution $u_{p}$ is given by

$$
u_{p}(x)=\frac{1}{N^{p^{\prime}} C_{N}^{p^{\prime} / N}} \int_{C_{N}|x|^{N}}^{C_{N} R^{N}} s^{\frac{p^{\prime}}{N}-p^{\prime}}\left(\int_{0}^{s} f^{*}(\sigma) d \sigma\right)^{\frac{1}{p-1}} d s,
$$

for almost every $x \in B_{R}$.

Our aim is to describe the behaviour of $u_{p}$ and the behaviour of $\left|\nabla u_{p}\right|^{p-2} \nabla u_{p}$ as $p$ goes to 1 .

We begin by introducing some notation. In what follows we will denote by

$$
\|f\|_{s}=\sup _{s \leq \sigma<C_{N} R^{N}} \sigma^{\frac{1}{N}} f^{* *}(\sigma), \quad \text { for every } s \in\left[0, C_{N} R^{N}[.\right.
$$

Clearly $\|f\|_{0} \equiv\|f\|_{L^{N, \infty}}$. Moreover we will denote by

$$
\text { (3.3) } s_{1}=\inf \left\{s \geq 0:\|f\|_{s} \leq N C_{N}^{\frac{1}{N}}\right\}, \quad s_{2}=\inf \left\{s \geq 0:\|f\|_{s}<N C_{N}^{\frac{1}{N}}\right\},
$$

and by $r_{1}$ and $r_{2}$ the radii of the balls centered in the origin having measure $s_{1}$ and $s_{2}$ respectively:

$$
C_{N} r_{1}^{N}=s_{1} \quad \text { and } \quad C_{N} r_{2}^{N}=s_{2} .
$$

We set $s_{1}=C_{N} R^{N}$ if the set $\left\{s \geq 0:\|f\|_{s} \leq N C_{N}^{\frac{1}{N}}\right\}$ is empty, and $s_{2}=C_{N} R^{N}$ if $\left\{s \geq 0:\|f\|_{s}<N C_{N}^{\frac{1}{N}}\right\}$ is empty. We explicitly remark that it results $s_{1} \leq s_{2}$ and hence $r_{1} \leq r_{2} \leq R$. Therefore the balls $B_{r_{1}}$ and $B_{r_{2}}$ centered at the origin and radii $r_{1}$ and $r_{2}$ respectively are both contained in $B_{R}$.

In general the limit of solutions $u_{p}$, as $p$ goes to 1 , is finite a.e. in $\Omega$ when the datum $f$ is small enough. For instance, if the datum $f$ is constant, that is $f^{*}(s)=\lambda>0$, then

$$
\begin{aligned}
& \lim _{p \rightarrow 1} u_{p}=0, \quad \text { if } \lambda \leq \frac{N}{R} ; \\
& \lim _{p \rightarrow 1} u_{p}=+\infty, \quad \text { if } \lambda>\frac{N}{R} ;
\end{aligned}
$$

(cf. [16]). In this section we analyze the behaviour of $u_{p}$ in a more general case, where $f$ is not constant, without any dependence on the 
smallness of the datum. Indeed Theorem 3.1 below states that, as $p$ goes to $1, u_{p}$ diverges in the ball $B_{r_{1}}$, that it has a finite non-negative limit (for which we give an upper bound) in the annulus $\overline{B_{r_{2}}} \backslash B_{r_{1}}$ of radii $r_{1}$ and $r_{2}$ and finally that it converges to zero in the annulus $B_{R} \backslash \overline{B_{r_{2}}}$ of radii $r_{2}$ and $R$.

Theorem 3.1. Let $u_{p}$ be the solution to problem (1.1). Then

$$
\begin{aligned}
& \lim _{p \rightarrow 1} u_{p}(x)=+\infty, \quad \text { for almost all } x \in B_{r_{1}}, \\
& 0 \leq \lim _{p \rightarrow 1} u_{p}(x) \leq R-|x| \quad \text { for almost all } x \in \overline{B_{r_{2}}} \backslash B_{r_{1}}, \\
& \lim _{p \rightarrow 1} u_{p}(x)=0, \quad \text { for almost all } x \in B_{R} \backslash \overline{B_{r_{2}}} \text {. }
\end{aligned}
$$

Remark 3.1. We explicitly observe that Theorem 3.1 improves the result proved in [10] where the behaviour of $u_{p}$ is studied just under a smallness assumption on $f$, i.e. $\|f\|_{L^{N, \infty}} \leq N C_{N}^{1 / N}$. Indeed if $\|f\|_{L^{N, \infty}}<N C_{N}^{1 / N}$, then $s_{1}=s_{2}=0$ and therefore we deduce that

$$
\lim _{p \rightarrow 1} u_{p}(x)=0 \quad \text { a.e. in } B_{R}
$$

if $\|f\|_{L^{N, \infty}}=N C_{N}^{1 / N}$, then $s_{1}=0$ and hence the limit function is a.e. finite in $B_{R}$, as in [10].

Remark 3.2. We point out that the values $r_{1}$ and $r_{2}$ (or equivalently $s_{1}$ and $s_{2}$ ) may be different. Indeed consider the function $f: B_{R} \rightarrow \mathbb{R}$ defined by $f(x)=\frac{N-1}{|x|}$. Then we have $f^{*}(\sigma)=C_{N}^{1 / N}(N-1) \sigma^{-1 / N}$ and $f^{* *}(\sigma)=N C_{N}^{1 / N} \sigma^{-\frac{1}{N}}$ for all $\left.\sigma \in\right] 0, C_{N} R^{N}$. Consequently $\|f\|_{s}=N C_{N}^{\frac{1}{N}}$ for every $s \in] 0, C_{N} R^{N}$ [; therefore, $s_{1}=0$ and $s_{2}=C_{N} R^{N}$. This example allows also to show that the value $R-|x|$ in (3.5) may be attained, i.e. then $\lim _{p \rightarrow 1} u_{p}(x)=R-|x|$ (see Remark 3.2 in $\left.[\mathbf{1 0}]\right)$.

Proof of Theorem 3.1: From (3.1) we can write, almost everywhere in $B_{r_{1}}$

$$
u_{p}(x)=U_{p}(x)+I_{p}
$$

where

(3.8) $U_{p}(x)=\frac{1}{N^{p^{\prime}} C_{N}^{p^{\prime} / N}} \int_{C_{N}|x|^{N}}^{C_{N} r_{1}^{N}} s^{\frac{p^{\prime}}{N}-p^{\prime}}\left(\int_{0}^{s} f^{*}(\sigma) d \sigma\right)^{\frac{1}{p-1}} d s, \quad$ a.e. $x \in B_{r_{1}}$,

$$
I_{p}=\frac{1}{N^{p^{\prime}} C_{N}^{p^{\prime} / N}} \int_{C_{N} r_{1}^{N}}^{C_{N} R^{N}} s^{\frac{p^{\prime}}{N}-p^{\prime}}\left(\int_{0}^{s} f^{*}(\sigma) d \sigma\right)^{\frac{1}{p-1}} d s
$$


Since $I_{p}$ is a nonnegative constant, by (3.7) it follows that

$$
u_{p}(x) \geq U_{p}(x)
$$

for almost all $x \in B_{r_{1}}$.

Now we evaluate $U_{p}(x)$, for almost every fixed $x \in B_{r_{1}}$. Denote by

$$
\sigma_{x}=C_{N}|x|^{N}
$$

Since $|x|<r_{1}$, it follows that $\sigma_{x}<s_{1}$. Thus, by definition (3.3) of $s_{1}$, we deduce that

$$
\|f\|_{\sigma_{x}}>N C_{N}^{\frac{1}{N}}
$$

By (3.2), there exists a constant $\hat{\sigma}_{x}$ such that

$$
0<\sigma_{x} \leq \hat{\sigma}_{x}<C_{N} R^{N}
$$

and

$$
\hat{\sigma}_{x}^{\frac{1}{N}} f^{* *}\left(\hat{\sigma}_{x}\right)>N C_{N}^{\frac{1}{N}} .
$$

Note also that (3.3) implies $\hat{\sigma}_{x}<s_{1}$. Hence, by the continuity of the function $g(\sigma)=\sigma^{\frac{1}{N}} f^{* *}(\sigma)$ and by $g\left(\hat{\sigma}_{x}\right)>N C_{N}^{\frac{1}{N}}$, it yields

$$
g(s)=s^{\frac{1}{N}} f^{* *}(s)>N C_{N}^{\frac{1}{N}},
$$

for every $s \in] \hat{\sigma}_{x}-\delta, \hat{\sigma}_{x}+\delta[$, for a suitable $\delta>0$.

Therefore,

$$
\begin{aligned}
U_{p}(x)= & \frac{1}{N^{p^{\prime}} C_{N}^{p^{\prime} / N}} \int_{C_{N}|x|^{N}}^{\hat{\sigma}_{x}} s^{\frac{p^{\prime}}{N}-p^{\prime}}\left(\int_{0}^{s} f^{*}(\sigma) d \sigma\right)^{\frac{1}{p-1}} d s \\
& +\frac{1}{N^{p^{\prime}} C_{N}^{p^{\prime} / N}} \int_{\hat{\sigma}_{x}}^{\hat{\sigma}_{x}+\delta} s^{\frac{p^{\prime}}{N}-p^{\prime}}\left(\int_{0}^{s} f^{*}(\sigma) d \sigma\right)^{\frac{1}{p-1}} d s \\
& +\frac{1}{N^{p^{\prime}} C_{N}^{p^{\prime} / N}} \int_{\hat{\sigma}_{x}+\delta}^{C_{N} r_{1}^{N}} s^{\frac{p^{\prime}}{N}-p^{\prime}}\left(\int_{0}^{s} f^{*}(\sigma) d \sigma\right)^{\frac{1}{p-1}} d s .
\end{aligned}
$$

Since the first and the third integral in (3.12) are non-negative, we obtain

$$
\begin{aligned}
U_{p}(x) & \geq \frac{1}{N^{p^{\prime}} C_{N}^{p^{\prime} / N}} \int_{\hat{\sigma}_{x}}^{\hat{\sigma}_{x}+\delta} s^{\frac{p^{\prime}}{N}-p^{\prime}}\left(\int_{0}^{s} f^{*}(\sigma) d \sigma\right)^{\frac{1}{p-1}} d s \\
& =\frac{1}{N C_{N}^{1 / N}} \int_{\hat{\sigma}_{x}}^{\hat{\sigma}_{x}+\delta} s^{\frac{1}{N}-1}\left(\frac{1}{N C_{N}^{1 / N}} s^{\frac{1}{N}-1} \int_{0}^{s} f^{*}(\sigma) d \sigma\right)^{\frac{1}{p-1}} d s .
\end{aligned}
$$


By (3.11) and definition of $f^{* *}$, we have

$$
\left.\frac{1}{N C_{N}^{1 / N}} s^{\frac{1}{N}-1} \int_{0}^{s} f^{*}(\sigma) d \sigma>1, \quad \forall s \in\right] \hat{\sigma}_{x}, \hat{\sigma}_{x}+\delta[.
$$

Thus, the right-hand side of (3.13) tends to $+\infty$ when $p$ goes to 1 and by (3.10) we deduce (3.4)

Now we prove (3.5). Using (3.1), we have

$$
u_{p}(x)=\frac{1}{N C_{N}^{1 / N}} \int_{C_{N}|x|^{N}}^{C_{N} R^{N}} s^{\frac{1}{N}-1}\left(\frac{s^{\frac{1}{N}-1}}{N C_{N}^{1 / N}} \int_{0}^{s} f^{*}(\sigma) d \sigma\right)^{\frac{1}{p-1}} d s
$$

for almost all $x \in \overline{B_{r_{2}}} \backslash B_{r_{1}}$, where, by (3.3),

$$
\frac{s^{\frac{1}{N}-1}}{N C_{N}^{1 / N}} \int_{0}^{s} f^{*}(\sigma) d \sigma \leq \frac{\|f\|_{s}}{N C_{N}^{1 / N}} \leq 1 \quad s \in\left[C_{N}|x|^{N}, C_{N} R^{N}[.\right.
$$

Hence immediately follows that $u_{p}$ is nonincreasing with respect to $p$; then $\lim _{p \rightarrow 1} u_{p}$ exists and it is non-negative. Furthermore, by (3.14) and (3.15), we get

$$
\begin{aligned}
u_{p}(x) & \leq \frac{1}{N C_{N}^{1 / N}} \int_{C_{N}|x|^{N}}^{C_{N} R^{N}} s^{\frac{1}{N}-1}\left(\frac{\|f\|_{s}}{N C_{N}^{1 / N}}\right)^{\frac{1}{p-1}} d s \\
& \leq \frac{1}{N C_{N}^{1 / N}} \int_{C_{N}|x|^{N}}^{C_{N} R^{N}} s^{\frac{1}{N}-1} d s .
\end{aligned}
$$

Therefore $\lim _{p \rightarrow 1} u_{p}(x) \leq R-|x|$, which gives (3.5).

Now we prove (3.6). By the definition of $s_{2}$ in (3.3) we deduce that

$$
\left.\|f\|_{s}<N C_{N}^{\frac{1}{N}}, \quad \forall s \in\right] s_{2}, C_{N} R^{N}[.
$$

Let $\hat{s} \in] s_{2}, C_{N} R^{N}$ [ be fixed. Since $\|f\|_{\hat{s}}<N C_{N}^{1 / N}$, we may write $\|f\|_{\hat{s}}=$ $(1-\epsilon) N C_{N}^{1 / N}$ for some $\epsilon>0$. Thus,

$$
s^{\frac{1}{N}-1} \int_{0}^{s} f^{*}(\sigma) d \sigma \leq(1-\epsilon) N C_{N}^{1 / N}
$$

for all $s \in[\hat{s}, R[$. Therefore by (3.1), we have

$$
\begin{aligned}
u_{p}(x) & \leq \frac{\left((1-\varepsilon) N C_{N}^{1 / N}\right)^{\frac{1}{p-1}}}{N^{p^{\prime}} C_{N}^{p^{\prime} / N}} \int_{C_{N}|x|^{N}}^{C_{N} R^{N}} s^{\frac{1}{N}-1} d s \\
& =\frac{(1-\varepsilon)^{\frac{1}{p-1}}}{N C_{N}^{1 / N}} \int_{C_{N}|x|^{N}}^{C_{N} R^{N}} s^{\frac{1}{N}-1} d s,
\end{aligned}
$$


for almost every $x \in B_{\hat{s}}$. Since

$$
\lim _{p \rightarrow 1} \frac{(1-\varepsilon)^{\frac{1}{p-1}}}{N C_{N}^{1 / N}}=0,
$$

we deduce that $\lim _{p \rightarrow 1} u_{p}(x)=0$ uniformly on $B_{\hat{s}}$. Thus (3.6) holds true.

Next we turn to describe the behaviour of $\left|\nabla u_{p}\right|^{p-2} \nabla u_{p}$ as $p$ goes to 1 .

Proposition 3.1. Let $u_{p}$ be the solution to problem (1.1). Denote by $z$ the vector field

$$
z=-\frac{f^{* *}\left(C_{N}|x|^{N}\right)}{N} x
$$

Then we have

(3.17) $\left|\nabla u_{p}(x)\right|^{p-2} \nabla u_{p}(x)=z(x)$, for every $p>1$ and for almost every $x \in B_{R}$.

Moreover it results

$$
\begin{aligned}
\|z\|_{L^{\infty}\left(B_{r_{1}} ; \mathbb{R}^{N}\right)} & >1, \\
\|z\|_{L^{\infty}\left(\overline{B_{r_{2}}} \backslash B_{r_{1}} ; \mathbb{R}^{N}\right)} & \leq 1, \\
\|z\|_{L^{\infty}\left(B_{R} \backslash \overline{B_{r_{2}}} ; \mathbb{R}^{N}\right)} & <1 .
\end{aligned}
$$

Proof: By (3.1) it follows that, for almost every $x \in B_{R}$,

$$
\begin{aligned}
\nabla u_{p}(x) & =-\left(\frac{|x|}{N} \frac{1}{C_{N}|x|^{N}} \int_{0}^{C_{N}|x|^{N}} f^{*}(\sigma) d \sigma\right)^{\frac{1}{p-1}} \frac{x}{|x|} \\
& =-\left(\frac{|x|}{N} f^{* *}\left(C_{N}|x|^{N}\right)\right)^{\frac{1}{p-1}} \frac{x}{|x|} .
\end{aligned}
$$

Hence, the vector field

$$
\left|\nabla u_{p}(x)\right|^{p-2} \nabla u_{p}(x)=-\frac{|x|}{N} f^{* *}\left(C_{N}|x|^{N}\right) \frac{x}{|x|},
$$

does not depend on $p$ and this implies (3.17). Moreover, since

$$
z(x)=-\frac{\left(C_{N}|x|^{N}\right)^{1 / N}}{N C_{N}^{1 / N}} f^{* *}\left(C_{N}|x|^{N}\right) \frac{x}{|x|},
$$

(3.18), (3.19) and (3.20) follow in a straightforward way from (3.2) and $(3.3)$. 
Remark 3.3. Let us point out that in the above proof, we have obtained

Therefore we deduce

$$
\left.\|z\|_{L^{\infty}\left(B_{R} \backslash B_{r} ; \mathbb{R}^{N}\right)}=\frac{\|f\|_{C_{N} r^{N}}}{N C_{N}^{1 / N}}, \quad \text { for all } r \in\right] 0, R[.
$$

$$
\|z\|_{\infty}=\sup _{0 \leq \sigma<C_{N} R^{N}} \frac{\sigma^{1 / N}}{N C_{N}^{1 / N}} f^{* *}(\sigma)=\frac{\|f\|_{N, \infty}}{N C_{N}^{1 / N}} .
$$

Remark 3.4. As a straightforward consequence of the definition of $z$, (3.1) becomes

$$
u_{p}(x)=\frac{1}{N C_{N}^{1 / N}} \int_{C_{N}|x|^{N}}^{C_{N} R^{N}} s^{\frac{1}{N}-1}\left|z\left(\frac{s^{1 / N}}{C_{N}^{1 / N}}\right)\right|^{\frac{1}{p-1}} d s
$$

for almost all $x \in B_{R}$. Thus, if $\|f\|_{L^{N, \infty}(\Omega)} \leq N C_{N}^{1 / N}$, then

$$
\lim _{p \rightarrow 1} u_{p}(x)=\frac{1}{N C_{N}^{1 / N}} \int_{C_{N}|x|^{N}}^{C_{N} R^{N}} s^{\frac{1}{N}-1} \chi_{\left\{\left|z\left(s^{1 / N} / C_{N}^{1 / N}\right)\right|=1\right\}}(s) d s
$$

and, changing the integration variable, we finally obtain that the above limit is equal to the measure of the set $\{|z(x)|=1\} \cap[|x|, R]$.

The above results, Theorem 3.1 and Proposition 3.1, allow to prove a stability type result for the "limit equation" (1.2). More precisely we will prove that if the norm of $f$ satisfies suitable smallness assumptions, then $u=\lim _{p \rightarrow 1} u_{p}$ is a solution to the "limit problem" which can be formally written

$$
\begin{cases}-\operatorname{div}\left(\frac{D u}{|D u|}\right)=f & \text { in } B_{R} \\ u=0 & \text { on } \partial B_{R}\end{cases}
$$

in the sense of the definition given in $[\mathbf{4}],[\mathbf{6}],[\mathbf{5}]$.

Theorem 3.2. Let $f \in L^{N, \infty}\left(B_{R}\right)$ with $\|f\|_{L^{N, \infty}} \leq N C_{N}^{1 / N}$ and let $u_{p}$ be the solution to problem (1.1), for any $1<p<\infty$. Then $u_{p}$ converges a.e. in $B_{R}$ to a function $u \in W_{0}^{1,1}\left(B_{R}\right)$ and there exists a vector field $z: B_{R} \rightarrow \mathbb{R}^{N}$ such that

$$
\begin{gathered}
z \in L^{\infty}\left(B_{R} ; \mathbb{R}^{N}\right) \text { with }\|z\|_{\infty} \leq 1 \\
\quad-\operatorname{div} z=f \text { in } \mathcal{D}^{\prime}\left(B_{R}\right) \\
z \cdot \nu \leq 0 \quad \mathcal{H}^{N-1} \text {-a.e. on } \partial B_{R}
\end{gathered}
$$

where $\nu$ denotes the outer normal to $\partial B_{R}$;

$$
z \cdot \nabla u=|\nabla u| \text { as measures in } B_{R} \text {. }
$$


Remark 3.5. We explicitly observe that the function $u$ given by Theorem 3.2 is a solution to (3.24) in the sense of the definition given in [4], [6], [5] (see Definition 4.1 and Remark 4.3 below).

Proof of Theorem 3.2: Since $\|f\|_{s} \leq N C_{N}^{1 / N}$ for all $\left.s \in\right] 0, C_{N} R^{N}$ [, we have $r_{1}=0$ and by Theorem 3.1 we have

$$
0 \leq u(x)=\lim _{p \rightarrow 1} u_{p}(x) \leq R-|x|, \quad \text { a.e. in } B_{R} .
$$

Moreover, from Proposition 3.1, we deduce that the vector field $z$ defined in (3.16) satisfies the conditions $\|z\|_{\infty} \leq 1$ and

$$
\int_{B_{R}}\left|\nabla u_{p}\right|^{p} d x \leq\left(\frac{\|f\|_{N, \infty}}{N C_{N}^{1 / N}}\right)^{p^{\prime}}\left|B_{R}\right| .
$$

Since $\|f\|_{N, \infty} \leq N C_{N}^{1 / N}$, Sobolev's inequality for $B V$-functions implies that

$$
\nabla u_{p} \rightarrow D u \quad \text { weakly* in the sense of measures. }
$$

On the other hand, by (3.16), (3.21) and (3.25), we have $u \in W_{0}^{1,1}\left(B_{R}\right)$ and

$$
\begin{array}{ll}
\nabla u(x)=0, & \text { if }|z(x)|<1, \\
\nabla u(x)=z(x)=-\frac{x}{|x|} & \text { if }|z(x)|=1 .
\end{array}
$$

Moreover from (3.17), since $u_{p}$ is a solution to problem (1.1), it follows that $-\operatorname{div} z=f$ in $\mathcal{D}^{\prime}\left(B_{R}\right)$. Furthermore, by definition of $z$, it results

$$
z(x)=-|z(x)| \frac{x}{|x|} \text {. }
$$

If $|x|=R$, then

$$
z \cdot \nu(x)=-|z(x)| \frac{x}{R} \cdot \frac{x}{R}=-|z(x)|<0
$$

Finally, a straightforward calculation shows that, for every Borel set $B \subset$ $B_{R}$

$$
\int_{B} z \cdot \nabla u=\int_{\{|z(x)|=1\} \cap B} z \cdot \nabla u=|\{|z(x)|=1\} \cap B|=\int_{B}|\nabla u| .
$$

This yields the conclusion.

Remark 3.6. One could think from the results in this section that the set where $|z| \leq 1$ is the same as the set where $|u|<+\infty$; this is not the case as the following example shows. 
Consider problem (1.1) with datum $f\left(C_{N}|x|^{N}\right)$ defined by

$$
f(s)= \begin{cases}0, & \text { if } 0 \leq s \leq C_{N}\left(\frac{R}{2}\right)^{N} \\ \frac{\lambda}{s^{1 / N}}, & \text { if } C_{N}\left(\frac{R}{2}\right)^{N}<s<C_{N} R^{N} .\end{cases}
$$

It is not difficult to check that the solution is given by

$$
u_{p}(x)=\frac{1}{N C_{N}^{1 / N}} \int_{C_{N} \max \left\{|x|, \frac{R}{2}\right\}^{N}}^{C_{N} R^{N}} s^{\frac{1}{N}-1} g(s)^{\frac{1}{p-1}} d s ;
$$

with

$$
g(s)=\frac{\lambda}{(N-1) C_{N}^{1 / N}}\left(1-\left(\frac{C_{N} R^{N}}{s 2^{N}}\right)^{1-\frac{1}{N}}\right), \quad C_{N} \frac{R^{N}}{2^{N}}<s<C_{N} R^{N} .
$$

Observe that $g$ is an increasing function; thus, if $\lambda \leq \frac{(N-1) C_{N}^{1 / N} 2^{N-1}}{2^{N-1}-1}$, then $g(s)<1$ for all $s<C_{N} R_{N}$ and so $u_{p}(x) \rightarrow 0$ everywhere in $B_{R}$. On the other hand, if $\lambda>\frac{(N-1) C_{N}^{1 / N} 2^{N-1}}{2^{N-1}-1}$, then $g(s)>1$ in an interval $] s_{0}, C_{N} R^{N}$ [, so that $u_{p}(x) \rightarrow+\infty$ everywhere.

Let us compute the vector field $z$ : Since

$$
\nabla u_{p}(x)= \begin{cases}0, & \text { if } 0 \leq|x| \leq \frac{R}{2} ; \\ -\frac{x}{|x|} g\left(C_{N}|x|^{N}\right)^{1 /(p-1)}, & \text { if } \frac{R}{2}<|x|<R ;\end{cases}
$$

and so $\left|\nabla u_{p}\right|^{p-2} \nabla u_{p}$ does not depend on $p$, it follows that

$$
z(x)= \begin{cases}0, & \text { if } 0 \leq|x| \leq \frac{R}{2} \\ -\frac{x}{|x|} g\left(C_{N}|x|^{N}\right), & \text { if } \frac{R}{2}<|x|<R\end{cases}
$$

If $\lambda \leq \frac{(N-1) C_{N}^{1 / N} 2^{N-1}}{2^{N-1}-1}$, then $|z|<1$ everywhere while if $\lambda>\frac{(N-1) C_{N}^{1 / N} 2^{N-1}}{2^{N-1}-1}$, then $|z|>1$ only in a neighborhood of the boundary that does not intersect $B_{R / 2}(0)$.

Hence, $B_{R / 2}(0) \subset\{|z| \leq 1\}$ but $\{|u|<+\infty\}=\emptyset$ for all $\lambda>$ $\frac{(N-1) C_{N}^{1 / N} 2^{N-1}}{2^{N-1}-1}$. Therefore, $\{|u|<+\infty\} \varsubsetneqq\{|z| \leq 1\}$ for these values. Finally, we observe that when the limit function blows up at the boundary, it blows up everywhere. 


\section{Stability results with $W^{-1, \infty}(\Omega)$ data}

Consider the nonlinear elliptic problems

$$
\begin{cases}-\operatorname{div}\left(\left|\nabla u_{p}\right|^{p-2} \nabla u_{p}\right)=\mu, & \text { in } \Omega ; \\ u_{p}=0, & \text { on } \partial \Omega\end{cases}
$$

where $p>1$ and the datum $\mu$ belongs to $W^{-1, \infty}(\Omega)$. Since by duality arguments, $W^{-1, \infty}(\Omega)$ is included in $W^{-1, p^{\prime}}(\Omega)$ for every $p>1$, then the existence and uniqueness of the solution $u_{p} \in W_{0}^{1, p}(\Omega)$ to problem (4.1) can be proved by classical methods (see, for instance, $[\mathbf{1 8}]$ ). In this section we will study the behaviour, as $p$ goes to 1 , of these solutions $u_{p}$ to problems (4.1). We prove that, if the norm in $W^{-1, \infty}(\Omega)$ of the datum $\mu$ is less than 1 , then $u_{p}$ converges to the function $u \equiv 0$; if the norm in $W^{-1, \infty}(\Omega)$ of the datum $\mu$ is equal to 1 , then $u_{p}$ converges to a function $u$ belonging to $B V(\Omega)$. Moreover we prove that $u_{p}$ does not converge to any $B V$-function if the norm in $W^{-1, \infty}(\Omega)$ of the datum $\mu$ is greater than 1 (see Subsection 4.1 below). As in the previous section, we deduce a stability result for the limit problem (1.2) when the norm in $W^{-1, \infty}(\Omega)$ of the datum $\mu$ is less than or equal to 1 . Actually we does not give a stability result in the case where $\|\mu\|_{W^{-1, \infty}(\Omega)}=1$ for all $\mu \in W^{-1, \infty}(\Omega)$. We study such a case when $\mu$ belongs to a subspace of $W^{-1, \infty}(\Omega)$, namely $\Gamma(\Omega)$, the predual space of $B V(\Omega)$ (see Subsection 4.2 below).

From now on, abusing of the terminology, we will say that $u_{p}$ is a sequence and we will consider subsequences of it, as $p$ goes to 1 .

\subsection{General data in $W^{-1, \infty}(\Omega)$.}

The main theorem of this subsection is the following.

Theorem 4.1. Let $u_{p}$ be the solution to problem (4.1).

If $\|\mu\|_{W^{-1, \infty}(\Omega)}<1$, then, as $p$ goes to 1 , $u_{p}$ converges to 0 in $L^{q}(\Omega)$, with $q<\frac{N}{N-1}$.

If $\|\mu\|_{W^{-1, \infty}(\Omega)}=1$, then, up to a subsequence, $u_{p}$ converges to a $B V$ function in $L^{q}(\Omega)$, with $q<\frac{N}{N-1}$.

If $\|\mu\|_{W^{-1, \infty}(\Omega)}>1$, then

$$
\lim _{p \rightarrow 1} \int_{\Omega}\left|\nabla u_{p}\right| d x=+\infty,
$$

and hence there is not any $u \in B V(\Omega)$ which is the weak* limit of $u_{p}$. 
Proof: Since $u_{p}$ is a weak solution to problem (4.1), the following inequalities hold true

$$
\begin{aligned}
\int_{\Omega}\left|\nabla u_{p}\right|^{p} d x & =\left\langle\mu, u_{p}\right\rangle_{W^{-1, \infty}(\Omega), W_{0}^{1,1}(\Omega)} \\
& \leq\|\mu\|_{W^{-1, \infty}(\Omega)} \int_{\Omega}\left|\nabla u_{p}\right| d x \\
& \leq\|\mu\|_{W^{-1, \infty}(\Omega)}|\Omega|^{1 / p^{\prime}}\left(\int_{\Omega}\left|\nabla u_{p}\right|^{p} d x\right)^{1 / p} .
\end{aligned}
$$

Therefore, one always has

$$
\int_{\Omega}\left|\nabla u_{p}\right|^{p} d x \leq\|\mu\|_{W^{-1, \infty}(\Omega)}^{p^{\prime}}|\Omega| .
$$

Assume first that $\|\mu\|_{W^{-1, \infty}(\Omega)}<1$. Thus, we can write $\|\mu\|_{W^{-1, \infty}(\Omega)}=$ $1-\epsilon$, for a suitable $\epsilon>0$. By Young inequality, we have

$$
\int_{\Omega}\left|\nabla u_{p}\right| d x \leq\left[\frac{1}{p}(1-\epsilon)^{\frac{p}{p-1}}+\frac{p-1}{p}\right]|\Omega| \leq|\Omega| .
$$

This estimate implies that there exist $u \in B V(\Omega)$ and a subsequence, still denoted by $u_{p}$, satisfying $u_{p} \rightarrow u$ in $L^{q}(\Omega)$, with $q<\frac{N}{N-1}$, and

$$
|D u|(\Omega)+\int_{\partial \Omega}|u| d \mathcal{H}^{N-1} \leq \liminf _{p \rightarrow 1} \int_{\Omega}\left|\nabla u_{p}\right| d x .
$$

By (4.4), letting $p \rightarrow 1$, we obtain

$$
|D u|(\Omega)+\int_{\partial \Omega}|u| d \mathcal{H}^{N-1}=0,
$$

and therefore $u=0$. Since $u \equiv 0$ is the unique limit point, by Sobolev inequality, we actually obtain that $\lim _{p \rightarrow 1} u_{p}=0$ in $L^{q}(\Omega)$, with $q<$ $\frac{N}{N-1}$.

Let us now assume that $\|\mu\|_{W^{-1, \infty}(\Omega)}=1$. Then (4.3) becomes $\int_{\Omega}\left|\nabla u_{p}\right|^{p} d x \leq|\Omega|$ and by Young's inequality we obtain

$$
\int_{\Omega}\left|\nabla u_{p}\right| d x \leq|\Omega|
$$


So that from Sobolev's inequality for $B V$-functions we deduce the existence of a function $u \in B V(\Omega)$ such that, up to subsequences,

$$
\begin{cases}\nabla u_{p} \rightarrow D u & \text { weakly* in the sense of measures, } \\ u_{p} \rightarrow u & \text { strongly in } L^{q}(\Omega), \quad 1 \leq q<\frac{N}{N-1} \\ u_{p} \rightarrow u & \text { a.e. in } \Omega .\end{cases}
$$

Finally let us assume that $\|\mu\|_{W^{-1, \infty}(\Omega)}>1$. Since $\|\mu\|_{W^{-1, \infty}(\Omega)}=$ $\lim _{p \rightarrow 1}\|\mu\|_{W^{-1, p^{\prime}(\Omega)}}$, we may take $\epsilon>0$ and $p_{0}>1$ such that $\|\mu\|_{W^{-1, p^{\prime}(\Omega)}}>$ $1+\epsilon$, for all $p \leq p_{0}$.

On the other hand, if $\varphi \in W_{0}^{1, p}(\Omega)$ with $\|\nabla \varphi\|_{L^{p}\left(\Omega ; \mathbb{R}^{N}\right)} \leq 1$, then

$$
\langle\mu, \varphi\rangle_{W^{-1, p^{\prime}}(\Omega), W_{0}^{1, p}(\Omega)}=\int_{\Omega}\left|\nabla u_{p}\right|^{p-1} \nabla u_{p} \cdot \nabla \varphi d x
$$

$$
\leq\left(\int_{\Omega}\left|\nabla u_{p}\right|^{p} d x\right)^{\frac{p-1}{p}} .
$$

Since by definition we have

$$
\|\mu\|_{W^{-1, p^{\prime}}(\Omega)}=\sup \left\{\langle\mu, \varphi\rangle_{W^{-1, p^{\prime}}(\Omega), W_{0}^{1, p}(\Omega)}: \int_{\Omega}|\nabla \varphi|^{p} d x \leq 1\right\},
$$

then (4.6) implies

$$
\|\mu\|_{W^{-1, p^{\prime}}(\Omega)}^{\frac{p}{p-1}} \leq \int_{\Omega}\left|\nabla u_{p}\right|^{p} d x
$$

Therefore,

$$
(1+\epsilon)^{\frac{p}{p-1}} \leq \int_{\Omega}\left|\nabla u_{p}\right|^{p} d x
$$

for $p \leq p_{0}$. This implies

$$
\lim _{p \rightarrow 1} \int_{\Omega}\left|\nabla u_{p}\right|^{p} d x=+\infty .
$$

The conclusion follows from

$$
\int_{\Omega}\left|\nabla u_{p}\right|^{p} d x=\left\langle\mu, u_{p}\right\rangle_{W^{-1, \infty}(\Omega), W_{0}^{1,1}(\Omega)} \leq\|\mu\|_{W^{-1, \infty}} \int_{\Omega}\left|\nabla u_{p}\right| d x .
$$

Let us prove the following result which describes the behaviour of $\left|\nabla u_{p}\right|^{p-2} \nabla u_{p}$. 
Proposition 4.1. Let $u_{p}$ be the solution to problem (4.1), then there exists a vector field $z \in L^{\infty}\left(\Omega ; \mathbb{R}^{N}\right)$ such that, up to subsequences,

$$
\begin{gathered}
\left|\nabla u_{p}\right|^{p-2} \nabla u_{p} \rightarrow z \quad \text { weakly in } L^{q}(\Omega) \text { for all } 1 \leq q<+\infty, \\
-\operatorname{div} z=\mu \text { in } \mathcal{D}^{\prime}(\Omega), \\
\|z\|_{\infty}=\|\mu\|_{W^{-1, \infty}(\Omega)} .
\end{gathered}
$$

Proof:

Step 1: Proof of (4.7): Arguing as in the proof of Theorem 4.1, we obtain inequality (4.3). Then for every $q, 1 \leq q<p^{\prime}$, we have

$$
\begin{aligned}
\int_{\Omega}\left|\nabla u_{p}\right|^{(p-1) q} & \leq\left(\int_{\Omega}\left|\nabla u_{p}\right|^{p}\right)^{(p-1) q / p}|\Omega|^{1-\frac{(p-1) q}{p}} \\
& \leq|\Omega| \frac{(p-1) q}{p}\|\mu\|_{W^{\prime-1, \infty}(\Omega)}^{p^{\prime}(p-1) q} \\
& =|\Omega| \|\left.\mu\right|_{W^{-1, \infty}(\Omega)} ^{q} .
\end{aligned}
$$

It yields that, for any $q$ fixed, the sequence $\left|\nabla u_{p}\right|^{p-2} \nabla u_{p}$ is bounded in $L^{q}\left(\Omega ; \mathbb{R}^{N}\right)$ and then there exists $z_{q} \in L^{q}\left(\Omega ; \mathbb{R}^{N}\right)$ such that, up to subsequences,

$$
\left|\nabla u_{p}\right|^{p-2} \nabla u_{p} \rightarrow z_{q} \quad \text { in } L^{q}(\Omega) \text { for all } 1 \leq q<+\infty .
$$

Moreover, by a diagonal argument we can find a limit $z$ that does not depend on $q$, that is

$$
\left|\nabla u_{p}\right|^{p-2} \nabla u_{p} \rightarrow z \quad \text { in } L^{q}(\Omega) \text { for all } 1 \leq q<+\infty .
$$

Now by (4.10) we deduce

$\left\|\left|\nabla u_{p}\right|^{p-2} \nabla u_{p}\right\|_{q} \leq|\Omega|^{1 / q}\|\mu\|_{W^{-1, \infty}(\Omega)} \quad$ for $1 \leq q<+\infty$ and for $\left.p \in\right] 1, q^{\prime}[$.

Therefore, by lower semicontinuity of the norm, we have

$$
\|z\|_{q} \leq|\Omega|^{1 / q}\|\mu\|_{W^{-1, \infty}(\Omega)} \text { for all } 1 \leq q<+\infty .
$$

Step 2: Proof of (4.8): Since $u_{p}$ is a distributional solution to problem (4.1), it follows that

$$
\int_{\Omega}\left|\nabla u_{p}\right|^{p-2} \nabla u_{p} \cdot \nabla \varphi d x=\langle\mu, \varphi\rangle_{W^{-1, \infty}(\Omega), W_{0}^{1,1}(\Omega)}, \quad \forall \varphi \in C_{0}^{\infty}(\Omega) .
$$

Hence, using (4.11) we obtain

$$
\int_{\Omega} z \cdot \nabla \varphi d x=\langle\mu, \varphi\rangle_{W^{-1, \infty}(\Omega), W_{0}^{1,1}(\Omega)}, \quad \forall \varphi \in C_{0}^{\infty}(\Omega),
$$

that is (4.8). 
Step 3: Proof of (4.9): For any fixed $h>0$ and $p>1$, we denote

$$
D_{p, h}=\left\{x \in \Omega:\left|\nabla u_{p}(x)\right|^{p-1}>h\right\} .
$$

By (4.10) for $q=1$, as $p$ goes to 1 we have

$$
\begin{aligned}
& \left|\nabla u_{p}\right|^{p-2} \nabla u_{p} \chi_{D_{p, h}} \rightarrow g_{h} \quad \text { weakly in } L^{1}\left(\Omega ; \mathbb{R}^{N}\right), \\
& \left|\nabla u_{p}\right|^{p-2} \nabla u_{p} \chi_{\Omega \backslash D_{p, h}} \rightarrow f_{h} \quad \text { weakly in } L^{1}\left(\Omega ; \mathbb{R}^{N}\right),
\end{aligned}
$$

for some $g_{h} \in L^{1}(\Omega)$ and $f_{h} \in L^{1}(\Omega)$. On the other hand by (4.10) with $q=1$

$$
\left|D_{p, h}\right| \leq \frac{1}{h} \int_{\Omega}\left|\nabla u_{p}\right|^{p-1} \leq \frac{|\Omega|\|\mu\|_{W^{-1, \infty}(\Omega)}}{h} .
$$

Therefore by Hölder's inequality and (4.15) for every fixed $\Phi \in L^{\infty}\left(\Omega ; \mathbb{R}^{N}\right)$, with $\|\Phi\|_{\infty} \leq 1$, we have

$(4.16)$

$$
\begin{aligned}
\left.\left|\int_{D_{p, h}}\right| \nabla u_{p}\right|^{p-2} \nabla u_{p} \cdot \Phi \mid & \leq\|\Phi\|_{\infty} \int_{D_{p, h}}\left|\nabla u_{p}\right|^{p-1} \\
& \leq\left|D_{p, h}\right|^{1-\frac{1}{q}}\left(\int_{\Omega}\left|\nabla u_{p}\right|^{(p-1) q}\right)^{\frac{1}{q}} \\
& \leq \frac{1}{h^{1-\frac{1}{q}}}|\Omega|^{1-\frac{1}{q}}\|\mu\|_{W^{-1, \infty}(\Omega)}^{1-\frac{1}{q}}|\Omega|^{\frac{1}{q}}\|\mu\|_{W^{-1, \infty}(\Omega)} \\
& \leq \frac{1}{h^{1-\frac{1}{q}}}|\Omega|\|\mu\|_{W^{-1, \infty}(\Omega)}^{2-\frac{1}{q}} .
\end{aligned}
$$

By (4.13) and (4.16), for any fixed $h>0$ we deduce

$$
\left|\int_{\Omega} g_{h} \cdot \Phi\right| \leq \frac{1}{h^{1-\frac{1}{q}}}|\Omega|\|\mu\|_{W^{-1, \infty}(\Omega)}^{2-\frac{1}{q}},
$$

for every $\Phi \in L^{\infty}\left(\Omega ; \mathbb{R}^{N}\right)$ such that $\|\Phi\|_{\infty} \leq 1$. By duality we deduce the following estimate for $g_{h}$

$$
\int_{\Omega}\left|g_{h}\right| \leq \frac{1}{h^{1-\frac{1}{q}}}|\Omega|\|\mu\|_{W^{-1, \infty}(\Omega)}^{2-\frac{1}{q}},
$$

for any fixed $h>0$. Moreover by definition of the set $D_{p, h}$,

$$
\left.\left|\chi_{\Omega \backslash D_{p, h}}\right| \nabla u_{p}\right|^{p-2} \nabla u_{p} \mid \leq h, \quad \text { a.e. in } \Omega .
$$


This implies, using the inequality contained in [24, p. 337], the following pointwise estimate for $f_{h}$

$$
\left|f_{h}\right| \leq h, \quad \text { a.e. in } \Omega,
$$

for any fixed $h>0$. Therefore $f_{h} \in L^{\infty}\left(\Omega ; \mathbb{R}^{N}\right)$. Applying once again (4.10), we have

$$
\int_{\Omega \backslash D_{p, h}}\left|\nabla u_{p}\right|^{q(p-1)} \leq \int_{\Omega}\left|\nabla u_{p}\right|^{q(p-1)} \leq|\Omega|\|\mu\|_{W^{-1, \infty}(\Omega)}^{q},
$$

that is, for some $q_{0}$,

$$
\begin{aligned}
\left\|\chi_{\Omega \backslash D_{p, h}}\left|\nabla u_{p}\right|^{p-2} \nabla u_{p}\right\|_{q} \leq|\Omega|^{1 / q}\|\mu\|_{W^{-1, \infty}(\Omega)} \\
\leq 2\|\mu\|_{W^{-1, \infty}(\Omega)} \text { for all } q \geq q_{0} .
\end{aligned}
$$

This implies

$$
\left\|f_{h}\right\|_{q} \leq 2\|\mu\|_{W^{-1, \infty}(\Omega)} \text { for all } q \geq q_{0} .
$$

Since $f_{h} \in L^{\infty}\left(\Omega ; \mathbb{R}^{N}\right)$, it yields

$$
\left\|f_{h}\right\|_{\infty} \leq 2\|\mu\|_{W^{-1, \infty}(\Omega)} .
$$

Therefore, for every $h>0$, we have

$$
z=f_{h}+g_{h}
$$

with

$$
\int_{\Omega}\left|g_{h}\right| \leq \frac{C_{q}}{h^{1-\frac{1}{q}}}
$$

and

$$
\left\|f_{h}\right\|_{\infty} \leq 2\|\mu\|_{W^{-1, \infty}(\Omega)}
$$

The above condition on $g_{h}$ gives

$$
\lim _{h \rightarrow \infty} g_{h}=0 \quad \text { in } L^{1}(\Omega)
$$

and hence

$$
\lim _{h \rightarrow \infty} f_{h}=\lim _{h \rightarrow \infty} z-g_{h}=z \quad \text { in } L^{1}(\Omega) .
$$

Since $\left\|f_{h}\right\|_{\infty} \leq 2\|\mu\|_{W^{-1, \infty}(\Omega)}$ for all $h>0$, we obtain that $z \in L^{\infty}\left(\Omega ; \mathbb{R}^{N}\right)$.

Then (4.12) implies

$$
\|z\|_{\infty} \leq\|\mu\|_{W^{-1, \infty}(\Omega)}
$$

From (4.8) and the definition of the norm $\|\mu\|_{W^{-1, \infty}(\Omega)}$, since we have

$$
\langle\mu, \varphi\rangle_{W^{-1, \infty}(\Omega), W_{0}^{1,1}(\Omega)}=\int_{\Omega} z \cdot \nabla \varphi \leq\|z\|_{\infty} \int_{\Omega}|\nabla \varphi|,
$$

the reverse inequality follows, and therefore (4.9) is proved. 
Remark 4.1. In Theorem 4.1, when $\|\mu\|_{W^{-1, \infty}(\Omega)}>1$, we did not state which is the pointwise limit of $u_{p}$. Nevertheless, an "a posteriori" argument can be done to obtain some kind of limit of the solutions. In fact, we can prove the following claim.

There exists a function $v$ satisfying, up to subsequences,

(4.17) $\left|u_{p}\right|^{p-1} \rightarrow v \quad$ weakly in $L^{q}(\Omega) \quad$ and $\quad\|v\|_{q} \leq|\Omega|^{1 / q}\|\mu\|_{W^{-1, \infty}(\Omega)}$, for all $1 \leq q<+\infty$.

To prove this claim, we must carefully apply Sobolev's inequality. It is well-known (see, for instance, [25, p. 57 or p. 82]) that a straightforward argument yields a simple connection between $S_{N, p}$ and $S_{N}$, namely: $S_{N, p} \leq \frac{(N-1) p}{N-p} S_{N},(1 \leq p<N)$ and so

$$
S_{N, p} \leq 2(N-1) S_{N} \quad \text { for } 1 \leq p \leq \frac{2 N}{3} .
$$

From (4.10), we deduce

$$
\left(\int_{\Omega}\left|\nabla u_{p}\right|^{q(p-1)}\right)^{\frac{1}{q(p-1)}} \leq|\Omega|^{\frac{1}{q(p-1)}}\|\mu\|_{W^{-1, \infty}(\Omega)}^{\frac{1}{p-1}} .
$$

Consider $r$ such that $1 \leq r(p-1) \leq \frac{2 N}{3}$, by applying Sobolev's inequality, we get

$$
\begin{aligned}
\left(\int_{\Omega}\left(\left|u_{p}\right|^{r(p-1)}\right)^{\frac{N}{N-r(p-1)}}\right)^{\frac{N-r(p-1)}{N r(p-1)}} & \leq S_{N, r(p-1)}\left(\int_{\Omega}\left|\nabla u_{p}\right|^{r(p-1)}\right)^{\frac{1}{r(p-1)}} \\
& \leq S_{N, r(p-1)}|\Omega|^{\frac{1}{r(p-1)}}\|\mu\|_{W^{-1, \infty}(\Omega)}^{\frac{1}{p-1}} .
\end{aligned}
$$

Since $\frac{N}{N-r(p-1)}>1$, Hölder's inequality implies

$$
\begin{aligned}
\left(\int_{\Omega}\left|u_{p}\right|^{r(p-1)}\right)^{\frac{1}{r(p-1)}} & \leq|\Omega|^{\frac{1}{N}}\left(\int_{\Omega}\left(\left|u_{p}\right|^{r(p-1)}\right)^{\frac{N}{N-r(p-1)}}\right)^{\frac{N-r(p-1)}{N r(p-1)}} \\
& \leq S_{N, r(p-1)}|\Omega|^{\frac{1}{r(p-1)}+\frac{1}{N}}\|\mu\|_{W^{-1, \infty}(\Omega)}^{\frac{1}{p-1}} .
\end{aligned}
$$

Therefore, taking $S_{N, r(p-1)} \leq 2(N-1) S_{N}$ into account, we obtain

$$
\left\|\left|u_{p}\right|^{p-1}\right\|_{r} \leq\left(2(N-1) S_{N}|\Omega|^{\frac{1}{r(p-1)}}+\frac{1}{N}\right)^{p-1}\|\mu\|_{W^{-1, \infty}(\Omega)},
$$

for all $r$ satisfying $1 \leq r(p-1) \leq \frac{2 N}{3}$. 
Now let $q$ satisfy $1 \leq q \leq \frac{1}{p-1}<r$ and apply Hölder's inequality, then

$$
\begin{aligned}
\left\|\left|u_{p}\right|^{p-1}\right\|_{q} & \leq|\Omega|^{\frac{1}{q}-\frac{1}{r}}\left(\int_{\Omega}\left|u_{p}\right|^{r(p-1)}\right)^{\frac{1}{r}} \\
& \leq|\Omega|^{\frac{1}{q}}\left(2(N-1) S_{N}|\Omega|^{\frac{1}{N}}\right)^{p-1}\|\mu\|_{W^{-1, \infty}(\Omega)} .
\end{aligned}
$$

Since $p \rightarrow 1$, it follows that we may consider any $q$ such that $1 \leq$ $q<+\infty$; moreover, $\left|u_{p}\right|^{p-1}$ is bounded in $L^{q}(\Omega)$ and its bound tends to $|\Omega|^{\frac{1}{q}}\|\mu\|_{W^{-1, \infty}(\Omega)}$ as $p \rightarrow 1$. Therefore, $(4.17)$ is a consequence of a diagonal argument.

We also remark that there is some connection between the functions $u=\lim _{p \rightarrow 1} u_{p}$ and $v$. Indeed, on the set $\{u=0\}$ it yields $v \leq \limsup _{p \rightarrow 1} e^{(p-1) \log \left|u_{p}\right|} \leq 1$ a.e., while on $\{|u|=+\infty\}$ we have $v \geq \liminf _{p \rightarrow 1} e^{(p-1) \log \left|u_{p}\right|} \geq 1$ a.e. Finally, up a null set, we obtain $v=1$ on $\{0<|u|<+\infty\}$.

Remark 4.2. Since the Marcinkiewicz space $L^{N, \infty}\left(B_{R}\right)$ is included in $W^{-1, \infty}\left(B_{R}\right)$, if $\Omega$ is the ball $B_{R}$ and the datum $\mu$ belongs to $L^{N, \infty}\left(B_{R}\right)$, then Proposition 4.1 and Theorem 4.1 hold true. Taking into account Proposition 4.1 and Remark 3.3, we may deduce that for every $f \in$ $L^{N, \infty}\left(B_{R}\right)$,

$$
\|f\|_{W-1, \infty\left(B_{R}\right)}=\sup _{0 \leq \sigma<C_{N} R^{N}} \frac{\sigma^{1 / N}}{N C_{N}^{1 / N}} f^{* *}(\sigma)=\frac{\|f\|_{N, \infty}}{N C_{N}^{1 / N}} .
$$

Observe that Theorem 3.1 implies the existence of a finite limit if and only if

$$
\|f\|_{N, \infty}=\sup _{0 \leq \sigma<C_{N} R^{N}} \sigma^{\frac{1}{N}} f^{* *}(\sigma) \leq N C_{N}^{\frac{1}{N}},
$$

that is, when $\|f\|_{W^{-1, \infty}\left(B_{R}\right)} \leq 1$.

As in the previous section, the study of the behaviour, as $p$ goes to 1 , of $u_{p}$ and $\left|\nabla u_{p}\right|^{p-2} \nabla u_{p}$ allows to deduce a stability result to the "limit problem" formally written

$$
\begin{cases}-\operatorname{div}\left(\frac{D u}{|D u|}\right)=\mu & \text { in } \Omega, \\ u=0 & \text { on } \partial \Omega .\end{cases}
$$

Let us begin by recalling the definition of solution to this problem (see [4], $[\mathbf{5}],[\mathbf{9}]$ and $[\mathbf{1 2}])$. To this aim, we need to introduce the following distribution (cf. [7]): 
Let $u$ be a function belonging to $B V(\Omega)$ and let $z$ be a vector field belonging to $L^{\infty}\left(\Omega ; \mathbb{R}^{N}\right)$ such that $\operatorname{div} z$, in the sense of distributions, belongs to $B V(\Omega)^{*}$, i.e.

$$
\langle\operatorname{div} z, \varphi\rangle=-\int_{\Omega} z \cdot \nabla \varphi d x
$$

for all $\varphi \in C_{0}^{\infty}(\Omega)$. Then we define the distribution

$$
(z, D u): C_{0}^{\infty}(\Omega) \rightarrow \mathbb{R}
$$

by

$$
\langle(z, D u), \varphi\rangle=-\int_{\Omega} u z \cdot \nabla \varphi d x-\langle\operatorname{div} z, u \varphi\rangle_{B V(\Omega)^{*}, B V(\Omega)},
$$

for every $\varphi \in C_{0}^{\infty}(\Omega)$. Since $u \in B V(\Omega) \subset L^{N^{\prime}, 1}(\Omega), \varphi \in C_{0}^{\infty}(\Omega)$, $z \in L^{\infty}\left(\Omega ; \mathbb{R}^{N}\right)$ and $\operatorname{div} z \in B V(\Omega)^{*}$, all terms in (4.19) make sense.

Moreover as in [5, pp. 126-127] we may define the weak trace of the exterior normal component of $z$, which will be denoted by $[z, \nu]$.

Definition 4.1. A function $u: \Omega \rightarrow \mathbb{R}$ is a solution to (4.18) if the following conditions hold true

$$
u \in B V(\Omega)
$$

there exists a vector field $z: \Omega \rightarrow \mathbb{R}^{N}$ such that

$$
\begin{gathered}
z \in L^{\infty}\left(\Omega ; \mathbb{R}^{N}\right) \text { with }\|z\|_{\infty} \leq 1 ; \\
-\operatorname{div} z=\mu \quad \text { in } \mathcal{D}^{\prime}(\Omega) ; \\
{[z, \nu] \in \operatorname{sign}(-u) \quad \mathcal{H}^{N-1} \text {-a.e. on } \partial \Omega ;} \\
(z, D u) \text { is a Radon measure and } \\
(z, D u)=|D u| \quad \text { as measures in } \Omega .
\end{gathered}
$$

Remark 4.3. Let us observe that if the function $u$ in the previous definition belongs to $W^{1,1}(\Omega)$, then the measure $(z, D u)$ coincides with $z \cdot \nabla u$. As already observed, this means that the function $u$ and the vector field $z$ whose existence has been proved in the previous section yields a solution to problem (3.24).

The announced stability result it is now an easy consequence of Theorem 4.1 and Proposition 4.1. 
Theorem 4.2. Let $u_{p}$ be the solution to problem (4.1).

If $\|\mu\|_{W^{-1, \infty}(\Omega)}<1$, then, as $p$ goes to $1, u_{p}$ converges to $u \equiv 0$ solution to problem (4.18) in the sense of Definition 4.1.

If $\|\mu\|_{W^{-1, \infty}(\Omega)}>1$, then there is not solution to problem (4.18) in the sense of Definition 4.1.

Remark 4.4. We explicitly remark that by Theorem 4.1 , if $\|\mu\|_{W^{-1, \infty}(\Omega)}=$ 1 , then $u_{p}$ converges, up to a subsequence, to a function $u \in B V(\Omega)$. Nevertheless we are not able to prove, in general, that $u$ is a solution to problem (4.18) in the sense of Definition 4.1 (cf. Examples 4.1 and 4.2 below).

We conclude this subsection by showing some examples with datum belonging to $W^{-1, \infty}(\Omega)$. The following example gives the explicit expression of the limit function $u$ when $\|\mu\|_{W^{-1, \infty}(\Omega)}=1$.

Example 4.1. Let $\Omega$ be an open subset in $\mathbb{R}^{N}$ containing $B_{R}(0)$ and consider a vector field $g: \Omega \rightarrow \mathbb{R}^{2}$ defined by

$$
g(x)= \begin{cases}-\frac{\sqrt{N}}{N}\left(\operatorname{sign} x_{1}, \ldots, \operatorname{sign} x_{N}\right), & \text { if }\left|x_{1}\right|+\cdots+\left|x_{N}\right| \leq R \\ 0, & \text { if }\left|x_{1}\right|+\cdots+\left|x_{N}\right|>R .\end{cases}
$$

Since $|g| \leq 1, \operatorname{div} g \in W^{-1, \infty}(\Omega)$ and $\|\operatorname{div}(g)\|_{W^{-1, \infty}(\Omega)}=1$. We point out that $\operatorname{div} g \notin L^{1}(\Omega)$ since evaluating this divergence some measures appear. The solution to

$$
\begin{cases}-\Delta_{p} u_{p}=\operatorname{div} g, & \text { in } \Omega ; \\ u_{p}=0, & \text { on } \partial \Omega ;\end{cases}
$$

is given by

$$
u_{p}(x)= \begin{cases}\frac{\sqrt{N} R}{N}-\frac{\sqrt{N}}{N}\left(\left|x_{1}\right|+\cdots+\left|x_{N}\right|\right), & \text { if }\left|x_{1}\right|+\cdots+\left|x_{N}\right| \leq R \\ 0, & \text { if }\left|x_{1}\right|+\cdots+\left|x_{N}\right|>R .\end{cases}
$$

Hence, $u_{p}$ does not depend on $p$ and

$$
\begin{aligned}
u(x) & =\lim _{p \rightarrow 1} u_{p}(x) \\
& = \begin{cases}\frac{\sqrt{N} R}{N}-\frac{\sqrt{N}}{N}\left(\left|x_{1}\right|+\cdots+\left|x_{N}\right|\right), & \text { if }\left|x_{1}\right|+\cdots+\left|x_{N}\right| \leq R ; \\
0, & \text { if }\left|x_{1}\right|+\cdots+\left|x_{N}\right|>R .\end{cases}
\end{aligned}
$$

It is easy to prove that $u$ is a non trivial solution to the limit problem. 
Example 4.2. Let us consider the problem

$$
\begin{cases}-\Delta_{p} u_{p}=\operatorname{div} g, & \text { in } B_{R} \\ u_{p}=0, & \text { on } \partial B_{R}\end{cases}
$$

where $g \in L^{\infty}\left(B_{R} ; \mathbb{R}^{N}\right)$ is a radial and bounded vector field. It is wellknown, see [23], that the solution of this problem is given by

$$
u_{p}(x)=\frac{1}{N C_{N}^{1 / N}} \int_{C_{N}|x|^{N}}^{C_{N} R^{N}} G(t)^{\frac{p^{\prime}}{p}} t^{\frac{1}{N}-1} d t,
$$

where $G$ is a non-negative function which depends on $u_{p}$ satisfying

$$
\int_{0}^{C_{N} R^{N}} G(t)^{p^{\prime}} d t=\int_{B_{R}}|g(x)|^{p^{\prime}} d x
$$

(see [2]). Thus, applying Hölder's inequality and performing easy computations, it follows that

$$
\begin{aligned}
u_{p}(x) \leq & \frac{1}{N C_{N}^{1 / N}}\left(\int_{0}^{C_{N} R^{N}} G(t)^{p^{\prime}} d t\right)^{1 / p}\left(\int_{C_{N}|x|^{N}}^{C_{N} R^{N}} t^{\frac{p^{\prime}}{N}-p^{\prime}} d t\right)^{1 / p^{\prime}} \\
\leq & \frac{1}{N C_{N}^{1 / N}} C_{N}^{1 / p} R^{N / p}\|g\|_{\infty}^{\frac{1}{p-1}} \\
& \times\left(\frac{N(p-1)}{N-p}\left(\left(C_{N}|x|^{N}\right)^{-\frac{N-p}{N(p-1)}}-\left(C_{N} R^{N}\right)^{-\frac{N-p}{N(p-1)}}\right)\right)^{1 / p^{\prime}} \\
= & \frac{R^{N / p}}{N}\|g\|_{\infty}^{\frac{1}{p-1}} \frac{1}{|x|^{\frac{N-p}{p}}}\left(\frac{N(p-1)}{N-p}\right)^{(p-1) / p}\left(1-\left(\frac{|x|}{R}\right)^{\frac{N-p}{p-1}}\right)^{(p-1) / p} \\
\leq & \frac{R^{N / p}}{N}\|g\|_{\infty}^{\frac{1}{p-1}} \frac{1}{|x|^{\frac{N-p}{p}}}\left(\frac{N(p-1)}{N-p}\right)^{(p-1) / p} .
\end{aligned}
$$

Therefore, if $\|g\|_{\infty}<1$, then $\|g\|_{\infty}^{1 /(p-1)}$ goes to 0 and so

$$
\lim _{p \rightarrow 1} u_{p}(x)=0 \text {. }
$$

On the other hand, if $\|g\|_{\infty}=1$, then

$$
0 \leq \lim _{p \rightarrow 1} u_{p}(x) \leq \frac{R^{N}}{N} \frac{1}{|x|^{N-1}} .
$$

(Observe that this estimate is worse than (3.5).) 


\subsection{Data in the predual space of $B V(\Omega)$.}

In the previous subsection, we have stated a stability result when $\|\mu\|_{W^{-1, \infty}(\Omega)} \neq 1$. The case $\|\mu\|_{W^{-1, \infty}(\Omega)}=1$ is the most interesting since then $\lim _{p \rightarrow \infty} u_{p}$ defines non-trivial solutions to the "limit problem" (4.18). To check that $\lim _{p \rightarrow \infty} u_{p}$ is indeed a solution to (4.18), apart from passing to the limit, some extension of Anzellotti's theory is required. We refer to the definition of solution to (4.18) given in Definition 4.1.

We are able to extend the Anzellotti theory in some distinguished subspaces of $W^{-1, \infty}(\Omega)$. The case of the space of all Guy David measures is cumbersome and will be provided in a forthcoming paper. The case of the predual space $\Gamma(\Omega)$ of $B V(\Omega)$ is shown in the Appendix. We point out (see Theorem 5.1 in the Appendix) that if $z \in L^{\infty}\left(\Omega ; \mathbb{R}^{N}\right)$ is a vector field such that its divergence in the sense of distributions belongs to $\Gamma(\Omega)$, then the distribution defined in (4.19) is always a Radon measure.

In this subsection we completely analyze the case where the datum $\mu$ belongs to $\Gamma(\Omega)$. The main theorem of this subsection is the following

Theorem 4.3. Let $\mu \in \Gamma(\Omega)$ and let $u_{p}$ be the solution to problem (4.1). Then the following statements are equivalent:

1) Up to subsequences, $u_{p}$ converges a.e., as $p$ goes to 1 , to a measurable function $u$ which is a solution to problem (4.18) in the sense of Definition 4.1.

2) $\|\mu\|_{W^{-1, \infty}(\Omega)} \leq 1$.

Remark 4.5. Since it is well-known that uniqueness does not hold to problem (4.18) (we refer, for instance, to [8, p. 485]), we cannot deduce that, when $\|\mu\|_{W^{-1, \infty}(\Omega)}=1$, there exists $\lim _{p \rightarrow 1} u_{p}$, but just that a "subsequence" converges, as stated.

Proof of Theorem 4.3: We start by assuming that $\|\mu\|_{W^{-1, \infty}(\Omega)} \leq 1$. By Proposition 4.1, there exists a vector field $z \in L^{\infty}\left(\Omega ; \mathbb{R}^{N}\right)$ such that $\|z\|_{\infty} \leq 1$ and

$$
\left|\nabla u_{p}\right|^{p-2} \nabla u_{p} \rightarrow z \quad \text { weakly in } L^{q}(\Omega) \text { for all } 1 \leq q<+\infty .
$$

Arguing as in the proof of Theorem 4.1, we obtain inequality (4.5) and then a function $u \in B V(\Omega)$ such that, up to subsequences,

$$
\begin{cases}\nabla u_{p} \rightarrow D u & \text { weakly* in the sense of measures, } \\ u_{p} \rightarrow u & \text { strongly in } L^{q}(\Omega), \quad 1 \leq q<\frac{N}{N-1} \\ u_{p} \rightarrow u & \text { a.e. in } \Omega .\end{cases}
$$


As a consequence, $u_{p} \rightarrow u$ weakly* in $B V(\Omega)$ and therefore

$$
\left\langle\mu, u_{p}\right\rangle_{\Gamma(\Omega), B V(\Omega)} \rightarrow\langle\mu, u\rangle_{\Gamma(\Omega), B V(\Omega)} .
$$

Since $u_{p}$ is a weak solution to problem (4.1) then, choosing as test function $u_{p} \varphi$ with $\varphi \in C_{0}^{\infty}(\Omega)$ and $\varphi \geq 0$, we get

$$
\int_{\Omega}\left|\nabla u_{p}\right|^{p} \varphi d x=\left\langle\mu, \varphi u_{p}\right\rangle_{\Gamma(\Omega), B V(\Omega)}-\int_{\Omega}\left|\nabla u_{p}\right|^{p-2} \nabla u_{p} \cdot \nabla \varphi u_{p} d x .
$$

Passing to the limit in the right hand side, since $\mu=-\operatorname{div} z$, we get

$$
\lim _{p \rightarrow 1} \int_{\Omega}\left|\nabla u_{p}\right|^{p} \varphi d x=\langle(z, D u), \varphi\rangle .
$$

On the other hand, by Young's inequality

$$
\int_{\Omega}\left|\nabla u_{p}\right| \varphi d x \leq \frac{1}{p} \int_{\Omega}\left|\nabla u_{p}\right|^{p} \varphi d x+\frac{p-1}{p} \int_{\Omega} \varphi d x
$$

and as a consequence,

$$
\int_{\Omega}|D u| \varphi \leq \liminf _{p \rightarrow 1} \int_{\Omega}\left|\nabla u_{p}\right| \varphi d x \leq \liminf _{p \rightarrow 1} \int_{\Omega}\left|\nabla u_{p}\right|^{p} \varphi d x .
$$

Therefore, by (4.28), it yields

$$
\int_{\Omega}|D u| \varphi \leq \int_{\Omega}(z, D u) \varphi
$$

for every $\varphi \in C_{0}^{\infty}(\Omega)$ with $\varphi \geq 0$. Hence, $|D u| \leq(z, D u)$ and $\|z\|_{\infty} \leq 1$ implies $|D u|=(z, D u)$, thus (4.24) is done.

Taking now $u_{p}$ as test function in the weak formulation of problem (4.1), it follows that

$$
\int_{\Omega}\left|\nabla u_{p}\right|^{p} d x=\left\langle\mu, u_{p}\right\rangle_{W^{-1, p^{\prime}}(\Omega), W_{0}^{1, p}(\Omega)}
$$

Applying Young's inequality we get

$$
\begin{aligned}
\int_{\Omega}\left|\nabla u_{p}\right| d x & \leq \frac{1}{p} \int_{\Omega}\left|\nabla u_{p}\right|^{p} d x+\frac{p-1}{p}|\Omega| \\
& =\frac{1}{p}\left\langle\mu, u_{p}\right\rangle_{\Gamma(\Omega), B V(\Omega)}+\frac{p-1}{p}|\Omega| .
\end{aligned}
$$

Now we let $p$ goes to 1 in (4.29), by (4.27), it yields

$$
|D u|(\Omega)+\int_{\partial \Omega}|u| d \mathcal{H}^{N-1} \leq\langle\mu, u\rangle_{\Gamma(\Omega), B V(\Omega)},
$$


or equivalently,

$$
-\langle\mu, u\rangle_{\Gamma(\Omega), B V(\Omega)}+\int_{\Omega}(z, D u)+\int_{\partial \Omega}|u| d \mathcal{H}^{N-1} \leq 0 .
$$

By Green's formula (5.4) in the Appendix below, we get

$$
\int_{\partial \Omega}[z, \nu] u d \mathcal{H}^{N-1}+\int_{\partial \Omega}|u| d \mathcal{H}^{N-1} \leq 0 .
$$

Since $\|[z, \nu]\|_{\infty} \leq\|z\|_{\infty} \leq 1$, it follows that

$$
[z, \nu] u+|u|=0 \quad \mathcal{H}^{N-1} \text {-a.e. on } \partial \Omega
$$

which proves $(4.22)$.

Now let us assume that $\|\mu\|_{W^{-1, \infty}(\Omega)}>1$. By Theorem 4.2 we deduce that there is not solution to problem (4.18).

Remark 4.6. We point out that the solution whose existence is proved in Theorem 4.3 satisfies a variational formulation, namely:

$$
\begin{aligned}
|D u|(\Omega)+ & \int_{\partial \Omega}|u| d \mathcal{H}^{N-1}+\langle\mu, w\rangle_{B V(\Omega)^{*}, B V(\Omega)} \\
& \leq \int_{\partial \Omega}|w| d \mathcal{H}^{N-1}+\int_{\Omega}(z, D w)+\langle\mu, u\rangle_{B V(\Omega)^{*}, B V(\Omega)}
\end{aligned}
$$

for every $w \in B V(\Omega)$.

Next, we will only sketch the proof of this fact, which is "inspired" in [5, pp. 133-134 and pp. 139-140].

Let $w \in W_{0}^{1,2}(\Omega)$ and take $w-u_{p}$ as test function in the weak formulation of problem (4.1) for $1<p \leq 2$, it follows that

$$
\int_{\Omega}\left|\nabla u_{p}\right|^{p-2} \nabla u_{p} \cdot \nabla w-\int_{\Omega}\left|\nabla u_{p}\right|^{p}=\left\langle\mu, w-u_{p}\right\rangle_{B V(\Omega)^{*}, B V(\Omega)} .
$$

In order to take limit when $p \rightarrow 1$, apply Young's inequality and have in mind (4.26) and (4.27), to get

$$
\begin{aligned}
|D u|(\Omega)+\int_{\partial \Omega}|u| d \mathcal{H}^{N-1}+\langle\mu, w\rangle_{B V(\Omega)^{*}, B V(\Omega)} & \\
& \leq \int_{\Omega} z \cdot \nabla w+\langle\mu, u\rangle_{B V(\Omega)^{*}, B V(\Omega)},
\end{aligned}
$$

for all $w \in W_{0}^{1,2}(\Omega)$. By approximating, this inequality holds for all $w \in W_{0}^{1,1}(\Omega)$. In the following step assume that $w \in W^{1,1}(\Omega)$, take into 
account $\left(w_{n}\right)_{n}$ (the sequence of Lemma 5.1), pass to the limit as $n \rightarrow \infty$ and obtain

$$
\begin{aligned}
|D u|(\Omega)+ & \int_{\partial \Omega}|u| d \mathcal{H}^{N-1}+\langle\mu, w\rangle_{B V(\Omega)^{*}, B V(\Omega)} \\
& \leq \int_{\partial \Omega}|w| d \mathcal{H}^{N-1}+\int_{\Omega} z \cdot \nabla w d x+\langle\mu, u\rangle_{B V(\Omega)^{*}, B V(\Omega)} .
\end{aligned}
$$

Finally, given $w \in B V(\Omega)$, by Proposition 5.1, consider $w_{n} \in W^{1,1}(\Omega)$ satisfying $\left.w_{n}\right|_{\partial \Omega}=\left.w\right|_{\partial \Omega}$ for all $n \in \mathbb{N}$,

$$
\begin{gathered}
w_{n} \rightarrow w \quad \text { in } L^{1}(\Omega), \\
\int_{\Omega}\left|\nabla w_{n}\right| d x \rightarrow|D w|(\Omega) .
\end{gathered}
$$

Next apply the above inequality to each $w_{n}$ and let $n$ goes to $\infty$ taking into account $\left\langle\mu, w_{n}\right\rangle_{B V(\Omega)^{*}, B V(\Omega)} \rightarrow\langle\mu, w\rangle_{B V(\Omega)^{*}, B V(\Omega)}$ and $\int_{\Omega} z$. $\nabla w_{n} d x \rightarrow \int_{\Omega}(z, D w)$. Then we arrive to the desired inequality (4.30).

Remark 4.7. The same scheme followed in Theorem 4.3 can be adapted when the datum lives in $L^{N}(\Omega)$. Indeed, if $\mu=f \in L^{N}(\Omega)$ and $\|f\|_{W^{-1, \infty}(\Omega)} \leq 1$, then (4.5) holds true and so we may find $u \in B V(\Omega)$ such that, up to subsequences,

$$
\begin{cases}\nabla u_{p} \rightarrow D u & \text { weakly* in the sense of measures, } \\ u_{p} & \text { is bounded in } L^{\frac{N}{N-1}}(\Omega) \\ u_{p} \rightarrow u & \text { a.e. in } \Omega .\end{cases}
$$

These two last properties imply that $u_{p} \rightarrow u$ weakly in $L^{\frac{N}{N-1}}(\Omega)$, so that (4.27) becomes

$$
\int_{\Omega} f u_{p} d x \rightarrow \int_{\Omega} f u d x
$$

Once we have obtained this convergence, we may follow the same proof of the above theorem and get that $u_{p}$ converges to a solution to problem (4.18) in the sense of Definition 4.1 if and only if $\|f\|_{W^{-1, \infty}(\Omega)} \leq 1$.

We point out that the above reasoning cannot be extended to all data belonging to the Marcinkiewicz space $L^{N, \infty}(\Omega)$ since, in general, $u_{p}$ bounded in $L^{\frac{N}{N-1}}, 1(\Omega)$ and $u_{p} \rightarrow u$ a.e. in $\Omega$ does not imply $u_{p} \rightarrow u$ weakly in $L^{\frac{N}{N-1}, 1}(\Omega)$. Nevertheless, we can handle every datum that belongs to $L^{N, \infty}(\Omega)$ by using truncation (see [19]). 
Example 4.3. In this example we give an element of the predual $\Gamma(\Omega)$ which does not belong to $L^{N, \infty}(\Omega)$.

Let $h \in C_{0}(]-1,1\left[^{N-1}\right)$ satisfy $0 \leq h \leq 1$ and $\left.h\right|_{[-1 / 2,1 / 2]^{N-1}} \equiv 1$. We consider $\Omega=]-1,1\left[^{N}\right.$ and $g: \Omega \rightarrow \mathbb{R}^{N}$ given by

$$
g\left(x_{1}, x_{2}, \ldots, x_{N}\right)=\left(\frac{\lambda}{1-\alpha}\left(1-\left|x_{1}\right|\right)^{1-\alpha} h\left(x_{2}, \ldots, x_{N}\right), 0,0, \ldots, 0\right),
$$

with $\lambda \in \mathbb{R}$ and $\frac{1}{N}<\alpha<1$, and we set $F \equiv \operatorname{div} g$.

Since $g \in C_{0}\left(\Omega ; \mathbb{R}^{N}\right)$, it follows that $F \in \Gamma(\Omega)$. On the other hand,

$$
F\left(x_{1}, x_{2}, \ldots, x_{N}\right)=\frac{-\lambda \operatorname{sign} x_{1}}{\left(1-\left|x_{1}\right|\right)^{\alpha}} h\left(x_{2}, \ldots, x_{N}\right) .
$$

We now prove that $F \notin L^{N, \infty}(\Omega)$. Indeed, given $t>0$, one has

$$
\begin{aligned}
& \left\{\left(x_{1}, x_{2}, \ldots, x_{N}\right) \in \mathbb{R}^{N}: \frac{\lambda}{\left(1-\left|x_{1}\right|\right)^{\alpha}} h\left(x_{2}, \ldots, x_{N}\right)>t\right\} \\
& \supset\left\{\left(x_{1}, x_{2}, \ldots, x_{N}\right) \in \mathbb{R}^{N}: \frac{\lambda}{\left(1-\left|x_{1}\right|\right)^{\alpha}}>t \text { and } h\left(x_{2}, \ldots, x_{N}\right)=1\right\} \\
& \supset\left\{x_{1} \in \mathbb{R}: \frac{\lambda}{\left(1-\left|x_{1}\right|\right)^{\alpha}}>t\right\} \times\left[\frac{-1}{2}, \frac{1}{2}\right]^{N-1} .
\end{aligned}
$$

Now, it follows from

$$
\left|\left\{x_{1} \in \mathbb{R}: \frac{\lambda}{\left(1-\left|x_{1}\right|\right)^{\alpha}}>t\right\}\right|=2\left(\frac{\lambda}{t}\right)^{1 / \alpha}
$$

that

$$
\left.\left|\left\{\left(x_{1}, x_{2}, \ldots, x_{N}\right) \in \mathbb{R}^{N}: \mid F x_{1}, x_{2}, \ldots, x_{N}\right)\right|>t\right\} \mid \geq \frac{C_{\lambda}}{t^{1 / \alpha}},
$$

with $1<\frac{1}{\alpha}<N$. By $(2.1)$, we deduce that $F \notin L^{N, \infty}(\Omega)$.

\section{Appendix}

This Appendix contains some simple facts on $\Gamma(\Omega)$ which we prove for the sake of completeness.

\subsection{Norm of $\Gamma(\Omega)$.}

We need the following result, which is stated in [7] (see also [5]).

Lemma 5.1. Given $u \in B V(\Omega)$, and so $\left.u\right|_{\partial \Omega} \in L^{1}(\partial \Omega)$, there exists a sequence $\left(w_{n}\right)_{n}$ in $W^{1,1}(\Omega) \cap C(\Omega)$ satisfying

(1) $\left.w_{n}\right|_{\partial \Omega}=\left.u\right|_{\partial \Omega}$. 
(2) $\int_{\Omega}\left|\nabla w_{n}\right| d x \leq \int_{\partial \Omega}|u| d \mathcal{H}^{N-1}+\frac{1}{n}$.

(3) $\int_{\Omega}\left|w_{n}\right| d x \leq \frac{1}{n}$

(4) $w_{n}(x)=0$, if $\operatorname{dist}(x, \partial \Omega)>\frac{1}{n}$.

A straightforward consequence of conditions (2) and (3) is

$$
w_{n} \rightarrow 0 \quad \text { weakly* in } B V(\Omega) \text {. }
$$

Proposition 5.1. If $\mu \in \Gamma(\Omega)$, then

$$
\|\mu\|_{W^{-1, \infty}(\Omega)}=\|\mu\|_{B V(\Omega)^{*}} .
$$

Proof: Since $\|\mu\|_{W^{-1, \infty}(\Omega)} \leq\|\mu\|_{B V(\Omega)^{*}}$, we only have to see the inequality $\|\mu\|_{B V(\Omega)^{*}} \leq\|\mu\|_{W^{-1, \infty}(\Omega)}$. To this end, let $u \in B V(\Omega)$ be such that

$$
|D u|(\Omega)+\int_{\partial \Omega}|u| d \mathcal{H}^{N-1} \leq 1
$$

and fix $\epsilon>0$. Given $u \in B V(\Omega)$, consider the sequence $\left(w_{n}\right)_{n}$ of Lemma 5.1; observe that (5.1) implies $\left\langle\mu, w_{n}\right\rangle \rightarrow 0$. Let $n \in \mathbb{N}$ satisfy

$$
\left|\left\langle\mu, w_{n}\right\rangle_{\Gamma(\Omega), B V(\Omega)}\right|+\frac{1}{n}<\epsilon .
$$

Since $n$ is already fixed and $\left.\left(u-w_{n}\right)\right|_{\partial \Omega}=0$, there exists a sequence $\left(\varphi_{k}\right)_{k}$ in $C_{0}^{\infty}(\Omega)$ such that $\varphi_{k} \rightarrow u-w_{n}$ weakly* in $B V(\Omega)$ and $\int_{\Omega}\left|\nabla \varphi_{k}\right| \leq \int_{\Omega}\left|D\left(u-w_{n}\right)\right|$. Hence,

$$
\left\langle\mu, \varphi_{k}\right\rangle_{\Gamma(\Omega), B V(\Omega)} \rightarrow\left\langle\mu, u-w_{n}\right\rangle_{\Gamma(\Omega), B V(\Omega)} \quad \text { as } k \rightarrow \infty
$$

and

$\int_{\Omega}\left|\nabla \varphi_{k}\right| d x \leq|D u|(\Omega)+\int_{\Omega}\left|\nabla w_{n}\right| \leq|D u|(\Omega)+\int_{\partial \Omega}|u| d \mathcal{H}^{N-1}+\frac{1}{n} \leq 1+\epsilon$.

Then

$$
\begin{aligned}
\left|\langle\mu, u\rangle_{\Gamma(\Omega), B V(\Omega)}\right| & \leq\left|\left\langle\mu, w_{n}\right\rangle_{\Gamma(\Omega), B V(\Omega)}\right|+\left|\left\langle\mu, u-w_{n}\right\rangle_{\Gamma(\Omega), B V(\Omega)}\right| \\
& \leq \epsilon+\lim _{k \rightarrow \infty}\left|\left\langle\mu, \varphi_{k}\right\rangle_{\Gamma(\Omega), B V(\Omega)}\right| \\
& \leq \epsilon+\lim _{k \rightarrow \infty}\left|\left\langle\mu, \varphi_{k}\right\rangle_{W^{-1, \infty}(\Omega), W_{0}^{1,1}(\Omega)}\right| \\
& \leq \epsilon+\liminf _{k \rightarrow \infty}\|\mu\|_{W^{-1, \infty}(\Omega)} \int_{\Omega}\left|\nabla \varphi_{k}\right| d x \\
& \leq \epsilon+\|\mu\|_{W^{-1, \infty}(\Omega)}(1+\epsilon) .
\end{aligned}
$$

Since $\epsilon$ is arbitrary, the conclusion follows. 


\subsection{The Anzellotti Theory in $\Gamma(\Omega)$.}

In this second part of the Appendix, we adapt Anzellotti's theory to the case $\operatorname{div}(z) \in \Gamma(\Omega)$. Recalling that $[z, \nu]$, the weak trace of the exterior normal component of $z$ is already considered (see [5, pp. 126-127]), we have to define $(z, D u)$, see that is a Radon measure for all $u \in B V(\Omega)$ and prove a Green's formula. We only show the proofs corresponding to $(z, D u)$ in Theorem 5.1 bellow, the others follow the same schema of $[7]$ adapted in the same way than the proof of Theorem 5.1.

Theorem 5.1. Let $z \in L^{\infty}\left(\Omega, \mathbb{R}^{N}\right)$ be a vector field such that its divergence in the sense of distributions $\xi=\operatorname{div}(z)$ belongs to $\Gamma(\Omega)$. Then (4.19) defines a Radon measure on $\Omega$ such that for every open set $U \subset \Omega$ and for every $\varphi \in C_{0}^{\infty}(U)$, we have

$$
|\langle(z, D u), \varphi\rangle| \leq\|\varphi\|_{\infty}\|z\|_{L^{\infty}(U)}|D u|(U) .
$$

Proof: Since $u \in B V(\Omega)$, we may find a sequence $\left(u_{n}\right)_{n}$ in $C^{\infty}(\Omega) \cap$ $B V(\Omega)$ such that

$$
\begin{gathered}
u_{n} \rightarrow u \text { in } L^{1}(\Omega), \\
\lim _{n \rightarrow \infty} \int_{V}\left|\nabla u_{n}\right|=|D u|(V),
\end{gathered}
$$

for all open set $V \subset \subset \Omega$ satisfying $|D u|(\partial V)=0$.

Now, given $\varphi \in C_{0}^{\infty}(U)$ take an open set $V$ such that $\operatorname{supp}(\varphi) \subset V \subset \subset$ $U$ and $|D u|(\partial V)=0$. We point out that $\left(u_{n} \varphi\right)_{n}$ is a sequence in $B V(\Omega)$ that weakly* converges to $u \varphi$. It follows from $\operatorname{div}(z)=\xi \in \Gamma(\Omega)$ that

$$
\left\langle\xi, u_{n} \varphi\right\rangle_{\Gamma(\Omega), B V(\Omega)} \rightarrow\langle\xi, u \varphi\rangle_{\Gamma(\Omega), B V(\Omega)} .
$$

Observe that $\int_{\Omega} z \cdot \nabla u_{n} \varphi d x=-\int_{\Omega} u_{n} z \cdot \nabla \varphi d x-\left\langle\xi, u_{n} \varphi\right\rangle_{\Gamma(\Omega), B V(\Omega)}$ for all $n \in \mathbb{N}$, and so the sequence $\left(\int_{\Omega} z \cdot \nabla u_{n} \varphi\right)_{n}$ converges to $\langle(z, D u), \varphi\rangle$. Since

$$
\left|\int_{\Omega} z \cdot \nabla u_{n} \varphi\right| d x \leq\|\varphi\|_{\infty}\|z\|_{L^{\infty}(U)} \int_{V}\left|\nabla u_{n}\right| d x
$$

letting $n$ goes to $+\infty$, we have

$$
|\langle(z, D u), \varphi\rangle| \leq\|\varphi\|_{\infty}\|z\|_{L^{\infty}(U)}|D u|(V) \leq\|\varphi\|_{\infty}\|z\|_{L^{\infty}(U)}|D u|(U) .
$$

It is straightforward consequence of the above arguments that

$$
\left|\int_{B}(z, D u)\right| \leq \int_{B}|(z, D u)| \leq\|z\|_{L^{\infty}(\Omega)}|D u|(B)
$$

for all Borel sets $B \subset \Omega$.

Finally, we state the Green formula that may be proved in our case. 
Theorem 5.2. Let $z \in L^{\infty}\left(\Omega, \mathbb{R}^{N}\right)$ be a vector field such that its divergence in the sense of distributions $\xi=\operatorname{div}(z)$ belongs to $\Gamma(\Omega)$. If $u \in B V(\Omega)$, then

$$
\langle\operatorname{div}(z), u\rangle_{\Gamma(\Omega), B V(\Omega)}+\int_{\Omega}(z, D u)=\int_{\partial \Omega}[z, \nu] u d \mathcal{H}^{N-1}
$$

Acknowledgements. This paper has been written during several visits of the first and third author to the University of Valencia and of the second author to the University of Naples "Federico II". We thank both universities for their warm hospitality. The authors would like to thank F. Andreu and J. M. Mazón for their interest in this work and for valuable discussions.

The second author acknowledges partial support by the Spanish project MTM2005-00620.

\section{References}

[1] A. Alvino, A limit case of the Sobolev inequality in Lorentz spaces, Rend. Accad. Sci. Fis. Mat. Napoli (4) 44 (1977), 105-112 (1978).

[2] A. Alvino and G. Trombetti, A class of degenerate nonlinear elliptic equations, (Italian), Ricerche Mat. 29(2) (1980), 193-212.

[3] L. Ambrosio, N. Fusco, And D. Pallara, "Functions of bounded variation and free discontinuity problems", Oxford Mathematical Monographs, The Clarendon Press, Oxford University Press, New York, 2000.

[4] F. Andreu, C. Ballester, V. Caselles, and J. M. Mazón, The Dirichlet problem for the total variation flow, J. Funct. Anal. 180(2) (2001), 347-403.

[5] F. Andreu, V. Caselles, and J. M. Mazón, "Parabolic quasilinear equations minimizing linear growth functionals", Progress in Mathematics 223, Birkhäuser Verlag, Basel, 2004.

[6] F. Andreu, J. M. Mazón, J. S. Moll, and V. Caselles, The minimizing total variation flow with measure initial conditions, Commun. Contemp. Math. 6(3) (2004), 431-494.

[7] G. Anzellotti, Pairings between measures and bounded functions and compensated compactness, Ann. Mat. Pura Appl. (4) 135 (1983), 293-318 (1984).

[8] G. Bellettini, V. Caselles, and M. Novaga, The total variation flow in $\mathbb{R}^{N}$, J. Differential Equations 184(2) (2002), 475-525. 
[9] G. Bellettini, V. Caselles, and M. Novaga, Explicit solutions of the eigenvalue problem $-\operatorname{div}\left(\frac{D u}{|D u|}\right)=u$ in $\mathbb{R}^{2}$, SIAM J. Math. Anal. 36(4) (2005), 1095-1129 (electronic).

[10] M. Cicalese And C. Trombetti, Asymptotic behaviour of solutions to $p$-Laplacian equation, Asymptot. Anal. 35(1) (2003), 27-40.

[11] F. Demengel, On some nonlinear equation involving the 1-Laplacian and trace map inequalities, Nonlinear Anal. 48(8), Ser. A: Theory Methods (2002), 1151-1163.

[12] F. Demengel, Functions locally almost 1-harmonic, Appl. Anal. 83(9) (2004), 865-896.

[13] L. C. Evans And R. F. GARIEPy, "Measure theory and fine properties of functions", Studies in Advanced Mathematics, CRC Press, Boca Raton, FL, 1992.

[14] A. FARINA, BV and Nikol'skiŭ spaces and applications to the Stefan problem, Atti Accad. Naz. Lincei Cl. Sci. Fis. Mat. Natur. Rend. Lincei (9) Mat. Appl. 6(3) (1995), 143-154.

[15] R. A. Hunt, On $L(p, q)$ spaces, Enseignement Math. (2) 12 (1966), 249-276.

[16] B. KawohL, On a family of torsional creep problems, J. Reine Angew. Math. 410 (1990), 1-22.

[17] B. KaWOHL, From $p$-Laplace to mean curvature operator and related questions, in: "Progress in partial differential equations: the Metz surveys", Pitman Res. Notes Math. Ser. 249, Longman Sci. Tech., Harlow, 1991, pp. 40-56.

[18] J. LERAY AND J.-L. LiOns, Quelques résultats de Višik sur les problèmes elliptiques nonlinéaires par les méthodes de Minty-Browder, Bull. Soc. Math. France 93 (1965), 97-107.

[19] A. Mercaldo, S. Segura de León, and C. Trombetti, Stability for renormalized solutions to $p$-Laplacian equations as $p$ goes to 1 , Preprint.

[20] Y. MEYER, "Oscillating patterns in image processing and nonlinear evolution equations", The fifteenth Dean Jacqueline B. Lewis memorial lectures, University Lecture Series 22, American Mathematical Society, Providence, RI, 2001.

[21] R. O'NeIL, Integral transforms and tensor products on Orlicz spaces and $L(p, q)$ spaces, J. Analyse Math. 21 (1968), 1-276.

[22] G. Talenti, Best constant in Sobolev inequality, Ann. Mat. Pura Appl. (4) 110 (1976), 353-372. 
[23] G. TALenti, Nonlinear elliptic equations, rearrangements of functions and Orlicz spaces, Ann. Mat. Pura Appl. (4) 120 (1979), $160-184$.

[24] A. C. ZaAnen, "Integration", Completely revised edition of "An introduction to the theory of integration", North-Holland Publishing Co., Amsterdam; Interscience Publishers John Wiley \& Sons, Inc., New York, 1967.

[25] W. P. Ziemer, "Weakly differentiable functions", Sobolev spaces and functions of bounded variation, Graduate Texts in Mathematics 120, Springer-Verlag, New York, 1989.

A. Mercaldo:

Dipartimento di Matematica e Applicazioni "R. Caccioppoli"

Università degli Studi di Napoli "Federico II"

Complesso Monte S. Angelo

via Cintia

80126 Napoli

Italy

E-mail address: mercaldo@unina.it

S. Segura de León:

Departament d'Anàlisi Matemàtica

Universitat de València

Dr. Moliner 50

46100 Burjassot, València

Spain

E-mail address: Sergio.Segura@uv.es

C. Trombetti:

Dipartimento di Matematica e Applicazioni "R. Caccioppoli"

Università degli Studi di Napoli "Federico II"

Complesso Monte S. Angelo

via Cintia

80126 Napoli

Italy

E-mail address: cristina@unina.it

Rebut el 7 de maig de 2007 . 